\title{
The Transient Behavior of processes in the TCP Paradigm
}

\author{
T.J. Ott ${ }^{*} \quad$ J.H.B. Kemperman ${ }^{\dagger}$
}

January 4, 2008

\begin{abstract}
This note derives the transient behavior of the stochastic process $X(t)$ of which in earlier papers it was proven that for $\alpha<1$ the process $(X(t))^{\frac{1}{1-\alpha}}$ (approximately, under low drop probability or ECN marking probability) describes the behavior of the congestion windows in certain Transport Protocols in the so-called TCP Paradigm.

The transient distribution is found explicitly, and is particularly transparent for moments like $E\left[(X(t))^{k} \mid X(0)\right]$. The purpose of this paper is to be part of the mathematical foundation when comparing the many protocols in the TCP Paradigm.
\end{abstract}

*Ott Associates, Chester, NJ. teun@teunisott.com

${ }^{\dagger}$ Department of Statistics, Rutgers University 


\section{Introduction}

In this paper we study the process $(X(t))_{-\infty<t<+\infty}$ obtained as follows: There is a Poisson Process with intensity $\lambda$ of which the points are denoted by $\left(\tau_{k}\right)_{k=-\infty}^{+\infty}$, and there is a number $0<c<1$. In between the points $\tau_{k}$ the process $X($.$) behaves as$

$$
\frac{d}{d t} X(t)=1
$$

and in the points $\tau_{k}$ we have

$$
X\left(\tau_{k}^{+}\right)=c X\left(\tau_{k}^{-}\right)
$$

If $\lambda=1$ we denote this process by $X^{*}(t)$.

Our interest in this process is due to its connection with existing and proposed Internet Transport Protocols. In [3] and [4] we proposed a class of potential such transport protocols, and made a first attempt to identify the more promising protocols in that class. The class contains classical TCP as well as Tom Kelly's Scalable TCP, see [10] and [11].

If the parameter $\beta$ in that class of protocols is chosen equal to one, and the drop probability $p$ or ECN marking probability $p$ is assumed to be constant, the protocols just referred to lead to the discrete time stochastic process $\left(W_{n}\right)_{n=0}^{\infty}$ as follows:

Let $\left(U_{n}\right)_{n=0}^{\infty}$ be independent, identically distributed random variables, each distributed uniformly $[0,1]$. Let $p$ be a probability, $0<p<1$. Define the i.i.d. random variables $\chi_{p, n}$ by

$$
\chi_{p, n}= \begin{cases}\text { success } & \text { if } U_{n} \geq p \\ \text { failure } & \text { if } U_{n}<p\end{cases}
$$

The process $W_{p, n}$ then is defined by

$$
W_{p, n+1}= \begin{cases}W_{p, n}+c_{1}\left(W_{p, n}\right)^{\alpha} & \text { if } \chi_{p, n}=\text { success } \\ \max \left(b W_{p, n}, \ell\right) & \text { if } \chi_{p, n}=\text { failure }\end{cases}
$$


where $\alpha<1, c_{1}>0,0<b<1, \ell \geq 0$. In classical TCP, $\alpha=-1, c_{1}=1, b=\frac{1}{2}$. In Scalable TCP, $\alpha=0$. In various versions of TCP $\ell$ usually is one MSS (Maximum Segment Size, i.e. maximum data size of a TCP packet) or a few MSSs. $p$ of course stands for the probability that a packet will be lost, or possibly marked in the Explicit Congestion Notification (ECN) mechanism. See [39, 40, 41] for a description of ECN.

In the limit for $p \downarrow 0$ the value of $\ell$ is not relevant, as long as it is constant.

$W_{p, n}$ stands for the congestion window after the $n$-th successful (or un-marked) acknowledgement.

The purpose of this paper is to be part of the mathematical foundation for a comparison of the many protocols in the TCP Paradigm. This includes finding the better combinations of $\alpha<1$ and $0<c<1$, but also includes study of desirable values of the "Slow Start Threshold" parameter. Such a study must also include the effects of a delay of 1 RTT in the feedback. A start on the latter is made in [8].

[6] and [7] prove stationarity of the process $W_{p, n}$. Curiously, if $(\ell=0$ and $\alpha<0)$ uniqueness of the stationary distribution has not been proven (yet?).

[6] proves that if $(\ell=0$ and $\alpha<0)$ then for this stationary distribution $E\left[\left(W_{p}\right)^{r}\right]<\infty$ if $-\frac{|\log (p)|}{|\log (b)|}<r<\frac{|\log (p)|}{|\alpha \log (b)|}$, else is infinite, while if $(\ell=0$ and $0 \leq \alpha<1) E\left[\left(W_{p}\right)^{r}\right]<\infty$ as long as $-\frac{|\log (p)|}{|\log (b)|}<r<\infty$, else is infinite. The same methods can be used to prove that if $\ell>0$ then $E\left[\left(W_{p}\right)^{r}\right]<\infty$ for all values of $r$, regardless of the value of $\alpha<1$.

[3] and [4] conjecture that for $p \downarrow 0$ the process $\frac{p}{c_{1}(1-\alpha)}\left(W_{p,\left\lfloor\frac{t}{p}\right\rfloor}\right)^{1-\alpha}$ converges weakly to the process $X^{*}(t)$ with $c=b^{1-\alpha}$, and that the stationary distributions correspondingly converge to the stationary distributions of the limiting process $X^{*}($.$) . In fact, [2], while$ not formulating the conjectures, uses them without proof.

These conjectures were proven in [7]. In addition, [5] obtains rate of convergence results for the convergence of the stationary distributions to the stationary distribution 
of the limiting process $X^{*}($.$) , see also below.$

The stationary distribution of the process $X($.$) was established in [2]. For example, if$ $Z$ has that stationary distribution it has the form

$$
Z=\sum_{k=0}^{\infty} c^{k} E_{k}
$$

where $\left(E_{k}\right)_{k=0}^{\infty}$ are independent, identically distributed random variables, all exponentially distributed with parameter $\lambda$, and for all (even complex) $r$,

$$
M(r)=E\left[Z^{r}\right]=\lambda^{-r} \Gamma(r+1) \prod_{k=1}^{\infty} \frac{\left(1-c^{r+k}\right)}{\left(1-c^{k}\right)} .
$$

If $r$ is a real integer this expression simplifies, see [2]. If $\lambda=1$ we denote $Z$ by $Z^{*}$ and $M(r)$ by $M^{*}(r)$.

In [5] it was investigated under what circumstances for the stationary distributions

$$
\lim _{p \downarrow 0} E\left[\left(\frac{p}{c_{1}(1-\alpha)}\left(W_{p}\right)^{1-\alpha}\right)^{r}\right]=M^{*}(r) .
$$

It was proven that if $(\ell=0$ and $\alpha \leq 0)$ then (1.7) holds for all $-\infty<r<+\infty$. If $(\ell=0$ and $0<\alpha<1)(1.7)$ was proven to hold for all $r \geq 0$. There is little doubt it also holds for $r<0$. The same methods can be used to prove that if $\ell>0$ then (1.7) holds for all $-\infty<r<+\infty$, regardless of the value of $\alpha$. [5] also contains rate of convergence results for (1.7).

In this paper we will obtain the transient behavior of the process $(X()$.$) , and thus$ for $p$ small the approximate transient behavior of the congestion window in Transport Protocols in the proposed class.

Mathematical analysis of TCP and TCP-like protocols has been an active area of research since 1996. [1] by now is of mostly historical interest. In addition to the ones already mentioned, notable papers are $[9,12,13,14,15,16,17,18,19,20,21,22,23$, 
$24,25,26,27,28,29,30]$. Of these, [30] contains an excellent review of the literature up to about 2004. [9] studies the transient behavior of a generalization of the process $X(t)$ where $Q_{k}=\frac{X\left(\tau_{k}^{+}\right)}{X\left(\tau_{k}^{-}\right)}$is random instead of deterministic, see also the remark following (3.10).

[7], while mostly a mathematical paper on the weak convergence issues for $p \downarrow 0$, contains some more information on the recent literature. In addition to the papers just mentioned, pride of ownership compels us to mention [31, 32, 33, 34, 35, 36, 37, 38].

It must be noted that the "time" used in this paper is packet-time, i.e. time is counted in data packets sent (or acknowledgements received). Several other papers studying Protocols in the TCP Paradigm use "clock-time", where time typically is counted in Round Trip Times (RTTs). If packet-time is denoted as $t_{P}$ and clock-time is denoted as $t_{C}$ we have

$$
d t_{P}=W d t_{C}
$$

where $W$ is the congestion window (in MSSs) at time $t_{P}$ (time $t_{C}$ ). The "packet-time stationary distribution" and the "clock-time stationary distribution" are of course not the same (in packet-time we sample faster when the congestion window is larger). [2] gives the relationship between the two stationary distributions: If $W_{P}$ and $W_{C}$ have respectively the "packet-time" stationary distribution and the "clock-time" stationary distribution then

$$
d P\left\{W_{P} \leq w\right\}=\frac{w d P\left\{W_{C} \leq w\right\}}{E\left[W_{C}\right]}, d P\left\{W_{C} \leq w\right\}=\frac{d P\left\{W_{P} \leq w\right\}}{w E\left[\frac{1}{W_{P}}\right]}
$$

Use of Clock Time with a constant (independent of the current Window Size) "probability per RTT" (e.g. "hazard rate") of a drop event leads to an interesting modeling issue: Implicitly it assumes a probability of drop per packet which is inversely proportional to the current window size $W$. This means that two or more TCP flows between the same source-destination pairs most of the time are assumed to have different drop probabilities per packet. 
The model described thus far assumes that the congestion window is the only constraint on the amount of data "in flight" (data sent but no acknowledgement received yet by the sender), that the congestion window is expressed in MSSs, that (practically) all datapackets contain one MSS worth of data, and that every individual data packet causes an acknowledgement to be sent. (No delayed acknowledgements.)

If desired, delayed acknowledgements can easily be incorporated into the model.

(1.8) indicates that we assume that the actual time (clock-time) between acknowledgement $n$ and acknowledgement $n+1$ can (away from error recovery etc) be assumed to be equal to $\frac{1}{W_{p, n}}$ of an RTT. To send $N$ packets, numbered $k+1, k+2, \cdots, k+N$ takes (assuming only a negligible amount of time is spent in Time-Outs and Recovery) about

$$
\frac{1}{W_{p, k}}+\frac{1}{W_{p, k+1}}+\cdots+\frac{1}{W_{p, k+N-1}} \text { RTTs. }
$$

(1.10) is of course a decent approximation only if $N$ is large. $N$ should be large compared with $\max _{k \leq j \leq k+N} W_{p, j}$.

We can condition on $W_{p . k}=w$ and take expected values. Thus we get that the expected amount of time to move MSSs $k+1, \cdots, k+N$ given $W_{p, k}=w$ is approximately

$$
E\left[\frac{1}{W_{p, k}}+\cdots+\frac{1}{W_{p, k+N-1}} \mid W_{p . k}=w\right] \text { RTTs. }
$$

Using the weak convergence results stated above, we see that with

$$
\rho=\frac{1}{1-\alpha}, \quad x=\frac{p w^{1-\alpha}}{c_{1}(1-\alpha)}
$$

the expected duration in (1.11) for $p$ small is approximately equal to

$$
\frac{p^{\frac{\alpha}{1-\alpha}}}{\left(c_{1}(1-\alpha)\right)^{\rho}} E\left[\int_{p k}^{p(k+N)} \frac{d t}{\left(X^{*}(t)\right)^{\rho}} \mid X^{*}(p k)=x\right] \text {. }
$$

For interesting results we should have that $p N$ is at least $\Omega(1)$. For a good understanding of (1.13) it is important to remember that for fixed $N$ (residual number of packets) $p N$ goes to zero when $p$ goes to zero. 
(1.13) also proves, again, a result known since 1996: the expected long range throughput for a large file, with $p$ small, should be approximately

$$
\left(\frac{c_{1}(1-\alpha)}{p}\right)^{\rho}\left(E\left[\left(Z^{*}\right)^{-\rho}\right]\right)^{-1} \mathrm{MSSs} / \mathrm{RTT},
$$

where $Z^{*}$ has the stationary distribution of the process $X^{*}($.$) . (1.6) thus can be used$ to compute the expected long range throughput. This results holds of course only if the assumptions of the model hold. For example, a home-user with a DSL modem will never get a throughput higher than the DSL link allows. Also, when there is a limit on the "flightsize" (e.g. receive window or send window) this leads to limits on the achievable throughput. On the other hand, (1.14) does not require that RTTs are constant.

This paper will not directly derive explicit expressions for entities like (1.13). It will derive explicit expressions for many transient entities such as $E\left[(X(t))^{k} \mid X(0)=x\right]$ and for entities like

$$
\begin{gathered}
\int_{0}^{\infty}\left(E\left[(X(t))^{r} \mid X(0)=x_{1}\right]-E\left[(X(t))^{r} \mid X(0)=x_{2}\right]\right) d t \\
\int_{0}^{\infty}\left(E\left[(X(t))^{r} \mid X(0)=x\right]-E\left[(X(t))^{r} \mid X(0)=Z\right]\right) d t
\end{gathered}
$$

where of course $Z$ has the stationary distribution as in (1.5) and is independent of the Poisson Process $\left(\tau_{k}\right)$, so that for all $t \geq 0(X(t) \mid X(0)=Z)$ has that stationary distribution, and for

$$
\frac{d}{d x} \int_{0}^{\infty}\left(E\left[(X(t))^{r} \mid X(0)=x\right]-E\left[(X(t))^{r} \mid X(0)=x_{0}\right]\right) d t
$$

In the sections $(3)-(7)$ the integrals $(1.15),(1.16),(1.17)$ will be re-defined as expected values of certain stochastic integrals. In the notation of those sections, (1.15) is denoted as $D\left(x_{1}, x_{2}, r, 0\right),(1.16)$ is denoted as $D\left(x_{1}, Z, r, 0\right)$ and (1.17) will be denoted as $\frac{d}{d x} D\left(x, x_{0}, r, 0\right)$. There will be a fourth parameter $(\beta)$, which is important in the derivations and which is zero in (1.15), (1.16), (1.17). 
With $\rho=\frac{1}{1-\alpha}, \frac{p^{\frac{\alpha}{1-\alpha}}}{\left(c_{1}(1-\alpha)\right)^{\rho}} D\left(x_{1}, x_{2},-\rho, 0\right)$ is the expected difference in duration until completion, in RTTs, for a (very) large file, between starting with an initial window $w_{1}=\left(\frac{c_{1}(1-\alpha) x_{1}}{p}\right)^{\rho}$ and starting with an initial window $w_{2}=\left(\frac{c_{1}(1-\alpha) x_{2}}{p}\right)^{\rho}$. Similarly, $\frac{p^{\frac{\alpha}{1-\alpha}}}{\left(c_{1}(1-\alpha)\right)^{\rho}} D(x, Z,-\rho, 0)$ describes the difference in expected duration for a large file, between starting with a window $w=\left(\frac{c_{1}(1-\alpha) x}{p}\right)^{\rho}$ and starting with a window in the stationary distribution. Since $-\rho<0, D(x, Z,-\rho, 0) \rightarrow-\infty$ if $x \rightarrow \infty$.

(1.17) similarly describes the marginal expected advantage, for ftping a very large file, of starting with a slightly larger congestion window.

For a large file consisting of $N$ packets (MSSs), if we start with the window in the stationary distribution, the expected time until completion is $N\left(\frac{p}{c_{1}(1-\alpha)}\right)^{\rho} E\left[\left(Z^{*}\right)^{-\rho}\right]$ RTTs. If we start with a window $w=\left(\frac{c_{1}(1-\alpha) x}{p}\right)^{\rho}$ the expected duration therefore will approximately be

$$
N\left(\frac{p}{c_{1}(1-\alpha)}\right)^{\rho} E\left[\left(Z^{*}\right)^{-\rho}\right]+\frac{p^{\frac{\alpha}{1-\alpha}}}{\left(c_{1}(1-\alpha)\right)^{\rho}} D(x, Z,-\rho, 0)
$$

RTTs. For $p$ (small, fixed) and $x$ fixed (1.18) becomes accurate if $N \rightarrow \infty$. If $x$ is of the order of $E\left[Z^{*}\right]$ it probably is enough that $N$ is large compared with $\frac{1}{p(1-c)}$. If $x$ is larger it probably is enough that $N$ is large compared with $\frac{1}{p}\left(\frac{1}{1-c}+\frac{\log (x)}{|\log (c)|}\right)$. These, and many others, are issues to be studied in a more engineering oriented paper.

The above may be useful, for example, in protocols using a "slow start - like" mechanisms, in choosing the analog of a "slow start threshold" parameter value.

Results will be obtained by two different methods: "elementary" methods and methods based on Laplace Transform techniques.

Section 2 contains some preliminaries. The next five Sections, $3-7$, use only elementary techniques to obtain results for various moments and related entities. Laplace Transform methods will be introduced in Section 8. Real domain results for transient densities of $(X(t) \mid X(0)=x)$ will be given in Section 9. Their proofs depend on Laplace 
Transforms from Section 8. Section 10 contains results for moments that could not be obtained by elementary methods but require the results from Section 9 .

Appendix A contains some results on special functions that will be used throughout this paper.

Appendix B derives a result needed in Section 10.

\section{Preliminaries}

Let $N(t)$ be the number of points of the Poisson Process in the time interval $(0, t]$. It is well known that

$$
P\{N(t)=k\}=\frac{(\lambda t)^{k}}{k !} e^{-\lambda t}
$$

One of the main objectives of this paper is to derive the joint distribution of $(N(t), X(t))$ given $X(0)=x$.

A special role is played by the process $X(t)$ with $X(0)=0$. We denote this process by $Y(t)$, and use the process $Y(t)$ to study the process $X(t)$, with of course the understanding that both processes are generated by the identical sequence $\left(\tau_{k}\right)$. With this understanding, we have the identity

$$
X(t)=\left(Y(t)+c^{N(t)} X(0)\right)
$$

which we often write as

$$
X(t)=\sum_{k=0}^{\infty}\left(Y(t)+c^{k} X(0)\right) \chi(N(t)=k) .
$$

It must be kept in mind that $Y(t)$ and $N(t)$ are (very!) dependent on each other, but of course independent of $X(0)$.

It is useful to observe that

$$
t c^{N(t)} \leq Y(t) \leq t,(t+x) c^{N(t)} \leq Y(t)+x c^{N(t)} \leq t+x c^{N(t)} \leq t+x
$$


for all $t \geq 0, x \geq 0$. This implies that for all $-\infty<\beta<+\infty$

$$
t^{r} e^{-\lambda t\left(1-c^{\beta+r}\right)} \leq E\left[(Y(t))^{r} c^{\beta N(t)}\right] \leq t^{r} e^{-\lambda t\left(1-c^{\beta}\right)}
$$

as long as $r \geq 0$ and

$$
t^{r} e^{-\lambda t\left(1-c^{\beta}\right)} \leq E\left[(Y(t))^{r} c^{\beta N(t)}\right] \leq t^{r} e^{-\lambda t\left(1-c^{\beta+r}\right)}
$$

as long as $r \leq 0$. It also shows that with probability 1 for every $0<\epsilon<\infty$ and $-\infty<\beta<+\infty$

$$
\int_{0}^{\epsilon}(Y(t))^{r} c^{\beta N(t)} d t \begin{cases}<\infty & \text { if } r>-1 \\ =\infty & \text { if } r \leq-1\end{cases}
$$

while as long as $x>0$ the similar integrals for $\left(Y(t)+x c^{N(t)}\right)^{r} c^{\beta N(t)}$ exist and are finite for all $r$, even complex $r$. Similar results hold for the integrals of $E\left[(Y(t))^{r} c^{\beta N(t)}\right]$ and $E\left[\left(Y(t)+x c^{N(t)}\right)^{r} c^{\beta N(t)}\right]$.

In Section 8 we will study transforms like

$$
\psi(x, t, s, z)=E\left[\exp \left\{-s\left(Y(t)+x c^{N(t)}\right)\right\} z^{N(t)}\right]
$$

Often, we write $z$ as $c^{\beta}$. In Section 9 we will use those transforms to obtain real domain information about transient densities. Before we proceed to transform techniques we restrict ourselves in the next five sections to elementary (real-domain) methods.

\section{$3 \quad$ Elementary methods for expected values}

A useful observation is that for any $r, \beta$,

$$
\frac{d}{d t} E\left[(X(t))^{r} c^{\beta N(t)}\right]=r E\left[(X(t))^{r-1} c^{\beta N(t)}\right]-\lambda\left(1-c^{r+\beta}\right) E\left[(X(t))^{r} c^{\beta N(t)}\right] .
$$

For $r=1$ this reduces to

$$
\frac{d}{d t} E\left[X(t) c^{\beta N(t)}\right]=e^{-\lambda t\left(1-c^{\beta}\right)}-\lambda\left(1-c^{1+\beta}\right) E\left[X(t) c^{\beta N(t)}\right]
$$


which yields

$$
E\left[X(t) c^{\beta N(t)}\right]=\left(X(0)-\frac{1}{\lambda c^{\beta}(1-c)}\right) e^{-\lambda t\left(1-c^{1+\beta}\right)}+\frac{e^{-\lambda t\left(1-c^{\beta}\right)}}{\lambda c^{\beta}(1-c)} .
$$

(3.1) with $r=2$ and (3.3) show that

$$
\begin{gathered}
E\left[(X(t))^{2} c^{\beta N(t)}\right]= \\
\frac{2}{\lambda^{2} c^{2 \beta}(1-c)\left(1-c^{2}\right)} \exp \left\{-\lambda t\left(1-c^{\beta}\right\}+\right. \\
\frac{2}{\lambda c^{1+\beta}(1-c)}\left(E[X(0)]-\frac{1}{\lambda c^{\beta}(1-c)}\right) \exp \left\{-\lambda t\left(1-c^{1+\beta}\right)\right\}+ \\
\left(E\left[(X(0))^{2}-\frac{2 E[X(0)]}{\lambda c^{1+\beta}(1-c)}+\frac{2}{\lambda^{2} c^{1+2 \beta}(1-c)\left(1-c^{2}\right)}\right) \exp \left\{-\lambda t\left(1-c^{2+\beta}\right)\right\} .\right.
\end{gathered}
$$

We easily see that in general, for $k$ a positive integer,

$$
E\left[(X(t))^{k} c^{\beta N(t)}\right]=\sum_{j=0}^{k} C_{j, k} \exp \left\{-\lambda t\left(1-c^{j+\beta}\right)\right\},
$$

where

$$
\begin{gathered}
C_{0,0}=1, \\
C_{j, k}=\frac{k !}{j ! \lambda^{k-j} c^{(k-j)(j+\beta)}(1-c)\left(1-c^{2}\right) \cdots\left(1-c^{k-j}\right)} C_{j, j} \text { if } 0 \leq j \leq k,
\end{gathered}
$$

(which if $j=k$ is a trivial identity), and

$$
\sum_{j=0}^{k} C_{j, k}=\sum_{j=0}^{k} \frac{k !}{j ! \lambda^{k-j} c^{(k-j)(j+\beta)}(1-c)\left(1-c^{2}\right) \cdots\left(1-c^{k-j}\right)} C_{j, j}=E\left[(X(0))^{k}\right] .
$$

We can re--write (3.7) as

$$
\sum_{j=0}^{k} \frac{1}{c^{(k-j) j}(1-c) \cdots\left(1-c^{k-j}\right)} \frac{\left(\lambda c^{\beta}\right)^{j} C_{j, j}}{j !}=\frac{\left(\lambda c^{\beta}\right)^{k} E\left[(X(0))^{k}\right]}{k !}
$$

to make it more obvious what infinite triangular matrix to invert. Using (3.8) and (3.6) we obtain

$$
\frac{\left(\lambda c^{\beta}\right)^{k} C_{k, k}}{k !}=\sum_{j=0}^{k} \frac{(-1)^{k-j}}{c^{j(k-j)+\frac{(k-j)(k-j-1)}{2}}(1-c)\left(1-c^{2}\right) \cdots\left(1-c^{k-j}\right)} \frac{\left(\lambda c^{\beta}\right)^{j} E\left[(X(0))^{j}\right]}{j !}=
$$




$$
\sum_{j=0}^{k} \frac{(-1)^{j}}{c^{j(k-j)+\frac{j(j-1)}{2}}(1-c)\left(1-c^{2}\right) \cdots\left(1-c^{j}\right)} \frac{\left(\lambda c^{\beta}\right)^{k-j} E\left[(X(0))^{k-j}\right]}{(k-j) !} .
$$

The proof of (3.9) amounts to inverting an infinite triangular matrix and uses (A.6) (with $\mu=k-1$ ) in Appendix A.

Substituting (3.9) into (3.6) and the results into (3.5) gives

$$
\begin{gathered}
\frac{\left(\lambda c^{\beta}\right)^{k} E\left[(X(t))^{k} c^{\beta N(t)}\right]}{k !}=\sum_{j=0}^{k} \frac{\left(\lambda c^{\beta}\right)^{j} E\left[(X(0))^{j}\right]}{j !} c^{-j(k-j)} \times \\
\left(\sum_{\nu=j}^{k} \frac{(-1)^{\nu-j} c^{\frac{1}{2}(\nu-j)(\nu-j-1)-(\nu-j)(k-j-1)} \exp \left\{-\lambda t\left(1-c^{\nu+\beta}\right)\right\}}{(1-c) \cdots\left(1-c^{k-\nu}\right) \cdot(1-c) \cdots\left(1-c^{\nu-j}\right)}\right) .
\end{gathered}
$$

(3.10) in the special case that $\beta=0$ is identical to Theorem 8 in [9] in the special case (in the language of that paper) that $P\{Q=c\}=1$.

(3.10) makes it easy to verify (again using (A.6)) that for $t \downarrow 0$ it reduces to $E\left[(X(0))^{k}\right]=$ $E\left[(X(0))^{k}\right]$.

Using Lemma A.2 in Appendix A, (3.10) can be re-written as

$$
\begin{gathered}
\frac{\lambda^{k}}{k !} E\left[(X(t))^{k} c^{\beta N(t)}\right]= \\
e^{-\lambda t} \sum_{\nu=0}^{k} \frac{\lambda^{\nu}}{\nu !} E\left[(X(0))^{\nu}\right] \sum_{\mu=0}^{\infty} \frac{(\lambda t)^{\mu+k-\nu}}{(\mu+k-\nu) !} c^{\mu(\nu+\beta)} \frac{\left(1-c^{\mu+1}\right) \cdots\left(1-c^{\mu+k-\nu}\right)}{(1-c) \cdots\left(1-c^{k-\nu}\right)} .
\end{gathered}
$$

Using (A.12) we can rewrite (3.11) as

$$
\begin{gathered}
\frac{\lambda^{k}}{k !} E\left[(X(t))^{k} c^{\beta N(t)}\right]= \\
e^{-\lambda t} \sum_{\nu=0}^{k} \frac{\lambda^{\nu}}{\nu !} E\left[(X(0))^{\nu}\right] \sum_{\mu=0}^{\infty} \frac{(\lambda t)^{\mu+k-\nu}}{(\mu+k-\nu) !} c^{\mu(\nu+\beta)} \frac{\left(1-c^{k-\nu+1}\right) \cdots\left(1-c^{k-\nu+\mu}\right)}{(1-c) \cdots\left(1-c^{\mu}\right)} .
\end{gathered}
$$

It sometimes is useful to write (3.12) as

$$
\frac{\lambda^{k}}{k !} E\left[(X(t))^{k} c^{\beta N(t)}\right]=
$$




$$
e^{-\lambda t} \sum_{\nu=0}^{k} \frac{\lambda^{\nu}}{\nu !} E\left[(X(0))^{\nu}\right] \sum_{\mu=0}^{\infty} \frac{(\lambda t)^{\mu+k-\nu}}{(\mu+k-\nu) !} c^{\mu \beta} \frac{\left(c^{\nu}-c^{k+1}\right) \cdots\left(c^{\nu}-c^{k+\mu}\right)}{(1-c) \cdots\left(1-c^{\mu}\right)} .
$$

In a formal sense we can write (3.13) as

$$
\begin{gathered}
\frac{\lambda^{k}}{k !} E\left[(X(t))^{k} c^{\beta N(t)}\right]= \\
e^{-\lambda t} \sum_{\nu=0}^{\infty} \frac{\lambda^{\nu}}{\nu !} E\left[(X(0))^{\nu}\right] \sum_{\mu=0}^{\infty} \frac{(\lambda t)^{\mu+k-\nu}}{\Gamma(\mu+k-\nu+1)} c^{\mu \beta} \frac{\left(c^{\nu}-c^{k+1}\right) \cdots\left(c^{\nu}-c^{k+\mu}\right)}{(1-c) \cdots\left(1-c^{\mu}\right)} .
\end{gathered}
$$

Namely, if $\nu \geq k+1$ then $\frac{1}{\Gamma(\mu+k-\nu+1)}=0$ if $\mu \leq \nu-k-1$ while the product in the inner sum equals 0 if $\mu \geq \nu-k$. In Section 10 we will see that as long as $X(0) \leq t$ we have, for all $r$, even complex:

$$
\begin{gathered}
\frac{\lambda^{r}}{\Gamma(r+1)} E\left[(X(t))^{r} c^{\beta N(t)}\right]= \\
e^{-\lambda t} \sum_{\nu=0}^{\infty} \frac{\lambda^{\nu}}{\nu !} E\left[(X(0))^{\nu}\right] \sum_{\mu=0}^{\infty} \frac{(\lambda t)^{\mu+r-\nu}}{\Gamma(\mu+r-\nu+1)} c^{\mu \beta} \frac{\left(c^{\nu}-c^{r+1}\right) \cdots\left(c^{\nu}-c^{r+\mu}\right)}{(1-c) \cdots\left(1-c^{\mu}\right)} .
\end{gathered}
$$

(3.15) converges absolutely as long as $X(0) \leq t$. It diverges if $P\{X(0)>t\}>0$.

In the special case that $X(0)=0(3.10)$ gives:

$$
\frac{\left(\lambda c^{\beta}\right)^{k} E\left[(Y(t))^{k} c^{\beta N(t)}\right]}{k !}=\sum_{\nu=0}^{k} \frac{(-1)^{\nu} c^{\frac{1}{2} \nu(\nu-1)-\nu(k-1)} \exp \left\{-\lambda t\left(1-c^{\nu+\beta}\right)\right\}}{(1-c) \cdots\left(1-c^{\nu}\right) \cdot(1-c) \cdots\left(1-c^{k-\nu}\right)},
$$

and similarly (3.12), or (3.16) with Lemma A2, gives

$$
\frac{\lambda^{k}}{k !} E\left[(Y(t))^{k} c^{\beta N(t)}\right]=e^{-\lambda t} \sum_{\mu=0}^{\infty} \frac{(\lambda t)^{\mu+k}}{(\mu+k) !} c^{\mu \beta} \frac{\left(1-c^{k+1}\right) \cdots\left(1-c^{k+\mu}\right)}{(1-c) \cdots\left(1-c^{\mu}\right)} .
$$

In Section 10 we will see that (3.17) can be generalized to the situation where $k$ is not a non-negative integer. For all $r$, even complex:

$$
\frac{\lambda^{r}}{\Gamma(r+1)} E\left[(Y(t))^{r} c^{\beta N(t)}\right]=e^{-\lambda t} \sum_{\mu=0}^{\infty} \frac{(\lambda t)^{\mu+r}}{\Gamma(\mu+r+1)} c^{\mu \beta} \frac{\left(1-c^{r+1}\right) \cdots\left(1-c^{r+\mu}\right)}{(1-c) \cdots\left(1-c^{\mu}\right)}
$$


We recognize (3.18) as (3.15) with $X(0)=0$. In fact, (3.18) will be proven before (3.15) and is used in the proof of (3.15).

By choosing $\beta=0$ we obtain the behavior of $E\left[(X(t))^{k}\right]$ and $E\left[(Y(t))^{k}\right]$, and (3.5) shows that the "Relaxation Time" of the process $X(t)$ is $\frac{1}{\lambda(1-c)}$ : If $X(0)$ is not too large compared with $E[Z]$, the time it takes for $X(t)$ to "reach the stationary distribution" is a moderate multiple of $\frac{1}{\lambda(1-c)}$.

(3.12) and (3.17) show that

$$
\begin{aligned}
& \frac{\lambda^{k+1}}{k !} \int_{0}^{\infty} E\left[\left(\left(Y(t)+x c^{N(t)}\right)^{k}-(Y(t))^{k}\right) c^{\beta N(t)}\right] d t= \\
& \sum_{\nu=1}^{k} \frac{(\lambda x)^{\nu}}{\nu !} \sum_{\mu=0}^{\infty} c^{\mu(\nu+\beta)} \frac{\left(1-c^{k-\nu+1}\right) \cdots\left(1-c^{k-\nu+\mu}\right)}{(1-c) \cdots\left(1-c^{\mu}\right)}
\end{aligned}
$$

The integral, and the sum, in (3.19) converge as long as $\operatorname{Re}(\beta)>-1$. More on convergence of this kind of integrals will be discussed in Sections 5, 6, 7. Using (A.2) (which in this case requires $\operatorname{Re}(\beta)>-1$ ) we re--write (3.19) as

$$
\begin{gathered}
\frac{\lambda^{k+1}}{k !} \int_{0}^{\infty} E\left[\left(\left(Y(t)+x c^{N(t)}\right)^{k}-(Y(t))^{k}\right) c^{\beta N(t)}\right] d t= \\
\sum_{\nu=1}^{k} \frac{(\lambda x)^{\nu}}{\nu !} \prod_{\mu=0}^{\infty} \frac{1-c^{k+\beta+1+\mu}}{1-c^{\nu+\beta+\mu}},
\end{gathered}
$$

which since $\nu \leq k$ and both are non-negative integers can be written as

$$
\begin{gathered}
\frac{\lambda^{k+1}}{k !} E\left[\int_{0}^{\infty}\left(\left(Y(t)+x c^{N(t)}\right)^{k}-(Y(t))^{k}\right) c^{\beta N(t)} d t\right]= \\
\sum_{\nu=1}^{k} \frac{(\lambda x)^{\nu}}{\nu !} \frac{1}{\left(1-c^{\nu+\beta}\right)\left(1-c^{\nu+\beta+1}\right) \cdots\left(1-c^{k+\beta}\right)} .
\end{gathered}
$$

(3.20) and (3.21 are identities as long as $\operatorname{Re}(\beta)>-1$. If $\beta$ is real and $\beta \leq-1$ the integrals are infinite but the sums in the RHSs are the analytic continuations of the expressions for $\operatorname{Re}(\beta)>-1$. As long as $\beta$ is real the interchange of expected value and integration is warranted by Fubini's theorem (all functions involved are non-negative). 
By using (3.21) twice, with different values of $x$, we obtain expressions like (1.15), however, with the constraint that $r$ must be a non-negative integer $k$. One of our goals is to generalize (3.21) to general $r$. It must be noted that in none of the three equations (3.19), (3.20), (3.21) we can change the upper limit of the sum from $k$ to $\infty$. Therefore, to find the entities described in (1.15), we must obtain new methods. This will be done in Sections 5, 6, 7 .

A second useful insight (beyond (3.1)) is that for any $r$

$$
\begin{gathered}
\frac{d}{d t} E\left[(X(t))^{r} \chi(N(t)=k)\right]= \\
\lambda c^{r} E\left[(X(t))^{r} \chi(N(t)=k-1)\right]+r E\left[(X(t))^{r-1} \chi(N(t)=k)\right]-\lambda E\left[(X(t))^{r} \chi(N(t)=k)\right] .
\end{gathered}
$$

For $k=0$ obviously

$$
E\left[(X(t))^{r} \chi(N(t)=0) \mid X(0)=x\right]=(x+t)^{r} \exp \{-\lambda t\}
$$

For $r=1(3.22)$ reduces to

$$
\begin{gathered}
\frac{d}{d t} E[X(t) \chi(N(t)=k)]= \\
\lambda c E[X(t) \chi(N(t)=k-1)]+P\{N(t)=k\}-\lambda E[X(t) \chi(N(t)=k)] .
\end{gathered}
$$

This yields

$$
E[X(t) \chi(N(t)=k) \mid X(0)=x]=\left(x \frac{(c \lambda t)^{k}}{k !}+\frac{\left(1-c^{k+1}\right)}{\lambda(1-c)} \frac{(\lambda t)^{k+1}}{(k+1) !}\right) e^{-\lambda t} .
$$

The proofs of the last few statements are left for the reader.

A third potentially useful insight is that for $m \in\{0,1,2, \cdots\}$

$$
\begin{gathered}
\left(\frac{d}{d x}\right)^{m} E\left[(X(t))^{r} c^{\beta N(t)} \mid X(0)=x\right]=\left(\frac{d}{d x}\right)^{m} E\left[\left(Y(t)+x c^{N(t)}\right)^{r} c^{\beta N(t)}\right]= \\
r(r-1) \cdots(r-m+1) E\left[\left(Y(t)+x c^{N(t)}\right)^{r-m} c^{(\beta+m) N(t)}\right] .
\end{gathered}
$$




\section{Stochastic Dominance and Bounds}

Let $X_{1}(t), X_{2}(t), \cdots$ be processes as in the previous Sections, with identical $(Y(t))_{t \geq 0}$ but different starting values $X_{1}(0), X_{2}(0), \cdots$, with of course $\left(X_{1}(0), X_{2}(0), \cdots\right)$ independent of $(Y(t))_{t \geq 0}$. By $(2.2)$, if $X_{1}(0)$ is (stochastically) less than $X_{2}(0)$ then, for all $t \geq 0, X_{1}(t)$ is (stochastically) less than $X_{2}(t)$.

Similarly, if $X_{3}(0)$ has the distribution of $Z$ in (1.5) then for all $t \geq 0 X_{3}(t)=$ $Y(t)+X_{3}(0) c^{N(t)}$ also has the distribution of $Z$.

Hence, if also $X_{1}(0)$ is stochastically less than $X_{3}(0)=Z$, and $Z$ is stochastically less than $X_{2}(0)$, then for all $t \geq 0 X_{1}(t)=Y(t)+X_{1}(0) c^{N(t)}$ is stochastically less than $Z$, and $Z$ is stochastically less than $X_{2}(t)=Y(t)+X_{2}(0) c^{N(t)}$.

We conjecture that in this case actually $X_{1}(t)=Y(t)+X_{1}(0) c^{N(t)}$ is stochastically increasing in $t$, and $X_{2}(t)=Y(t)+X_{2}(0) c^{N(t)}$ is stochastically decreasing in $t$, in both cases of course with the distribution of $Z$ as limit distribution (for $t \uparrow \infty$ ). Thus far we have been unable to prove this conjecture.

However, the following is enough for the needs of this paper:

Lemma $1(Y(t))_{t \geq 0}$ is stochastically increasing in $t$, with for limit distribution $(t \uparrow \infty)$ the distribution of $Z$.

Proof: Let $0<t_{1}<t_{2}$. Then

$$
Y\left(t_{2}\right)=Z\left(t_{2}-t_{1}\right)+Y\left(t_{1}\right) c^{N\left(t_{2}\right)-N\left(t_{1}\right)}>Z\left(t_{2}-t_{1}\right)
$$

where $Z\left(t_{2}-t_{1}\right)$ has the same distribution as $Y\left(t_{2}-t_{1}\right)$. Hence, $Y\left(t_{2}\right)$ is stochastically larger than $Y\left(t_{2}-t_{1}\right)$ for all $0<t_{1}<t_{2}$. This proves the lemma.

Clearly, if $r>0$ then $E\left[(Y(t))^{r}\right]$ increases from 0 at $t=0$ to $M(r)$ at $t=\infty$, and if $r<0$ then $E\left[(Y(t))^{r}\right]$ decreases from $+\infty$ at $t=0$ to $M(r)$ at $t=\infty$. More quantitatively: 
If $r \geq 0$ then

$$
t^{r} e^{-\lambda t\left(1-c^{r}\right)} \leq E\left[\left(Y(t)^{r}\right] \leq \min \left(M(r), t^{r}\right)\right.
$$

while if $r \leq 0$

$$
\max \left(M(r), t^{r}\right) \leq E\left[(Y(t))^{r}\right] \leq t^{r} e^{\lambda t\left(c^{r}-1\right)}
$$

and in both cases

$$
\lim _{t \rightarrow \infty} E\left[(Y(t))^{r}\right]=M(r)
$$

For the next result we need an intermediate step:

Observation If $U$ and $V$ are non-negative random variables and $\eta>0, r \geq 0$, then

$$
\begin{gathered}
E\left[(U+V)^{r}\right]=E\left[(U+V)^{r} \chi(V<\eta U)\right]+E\left[(U+V)^{r} \chi\left(U \leq \frac{1}{\eta} V\right)\right] \leq \\
(1+\eta)^{r} E\left[U^{r}\right]+\left(\frac{1+\eta}{\eta}\right)^{r} E\left[V^{r}\right] .
\end{gathered}
$$

The result still holds if $r \leq 0$. (The proof requires a trivial modification.) However, in that case it is not of much use in the following:

Lemma 2 Let $X(0)$ be random (non-negative), independent of $(Y(t))_{t \geq 0}$. If now $r \geq 0$ then for all $\eta(t)>0$

$$
\begin{gathered}
\max \left(E\left[(Y(t))^{r}\right], E\left[(X(0))^{r}\right] e^{-\lambda t\left(1-c^{r}\right)}\right) \leq E\left[\left(Y(t)+X(0) c^{N(t)}\right)^{r}\right] \leq \\
(1+\eta(t))^{r}\left(E\left[(Y(t))^{r}\right]+\left(\frac{1+\eta(t)}{\eta(t)}\right)^{r} E\left[(X(0))^{r}\right] e^{-\lambda t\left(1-c^{r}\right)}\right),
\end{gathered}
$$

while if $r \leq 0$ then

$$
E\left[\left(Y(t)+X(0) c^{N(t)}\right)^{r}\right] \leq \min \left(E\left[(Y(t))^{r}\right], E\left[(X(0))^{r}\right] e^{\lambda t\left(c^{r}-1\right)}\right)
$$

In (4.6) we can choose (for example) $\eta(t)=e^{-\frac{\lambda t}{2 r}\left(1-c^{r}\right)}$ to get a bound on how fast $E\left[\left(Y(t)+X(0) c^{N(t)}\right)^{r}\right]$ approaches $E\left[(Y(t))^{r}\right]$ if $t \rightarrow \infty$. By substituting (4.2), (4.3) into (4.6), (4.7) and using (4.4) we easily prove 
Theorem 1A For all $0<\epsilon<\infty, 0 \leq x<\infty,-\infty<r<+\infty$, the integral

$$
\int_{\epsilon}^{\infty}\left(Y(t)+x c^{N(t)}\right)^{r} c^{\beta N(t)} d t
$$

is finite with probability 1 , and has finite expected value, as long as $\beta>0$. If $\beta \leq 0$ the integral, with probability 1 , is infinite.

Clearly, in Theorem 1A we can allow $\beta, r$ to be complex valued. If $\operatorname{Re}(\beta)>0$ then for all $r$ the integral converges with probability one, and has a well defined expected value. If $\operatorname{Re}(\beta) \leq 0$ then for all $r$ the integral does not converge.

Theorem 1B Unless $(x=0$ and $\operatorname{Re}(r) \leq-1)$ the integral

$$
\int_{0}^{\epsilon}\left(Y(t)+x c^{N(t)}\right)^{r} c^{\beta N(t)} d t
$$

$(0<\epsilon<\infty, 0 \leq x<\infty,-\infty<r<+\infty,-\infty<\beta<+\infty)$ is finite with probability 1 and has finite expected value. If simultaneously $x=0$ and $R e(r) \leq-1$ the integral diverges with probability 1. (See also (2.7).)

Proof: Considering the results earlier in this section, the only statement which still needs proof is that if $\beta$ is real and $\beta \leq 0$ the integral in (4.8) is infinite with probability one. Because of the construction of the process $(X(t))$ convergence of the integral (4.8) requires that $\lim _{t \rightarrow \infty}(X(t))^{r} c^{\beta N(t)}=0$. This clearly has probability zero if $\operatorname{Re}(\beta) \leq 0$.

Paraphrasing Theorem 1: The critical point $t=\infty$ leads to divergence if and only if $\operatorname{Re}(\beta) \leq 0$. The critical point $t=0$ leads to divergence if and only if $(x=0$ and $\operatorname{Re}(r) \leq-1)$.

We see

Lemma 3 If $\operatorname{Re}(\beta)>0$ and $x>0$ the expected value

$$
E\left[\int_{0}^{\infty}\left(Y(t)+x c^{N(t)}\right)^{r} c^{\beta N(t)} d t\right]
$$


is analytic in $r$ on the whole complex plane, while if $\operatorname{Re}(\beta)>0$ and $x=0$ it is analytic in $r$ on the halfplane $\operatorname{Re}(r)>-1$.

Lemma 4 Similarly, if $\operatorname{Re}(\beta)>-1$ and $x>0$

$$
\frac{d}{d x} E\left[\int_{0}^{\infty}\left(Y(t)+x c^{N(t)}\right)^{r} c^{\beta N(t)} d t\right]=r E\left[\int_{0}^{\infty}\left(Y(t)+x c^{N(t)}\right)^{r-1} c^{(\beta+1) N(t)} d t\right]
$$

is an entire function of $r$, and hence

$$
\begin{gathered}
\int_{0}^{\infty}\left(\left(Y(t)+x_{1} c^{N(t)}\right)^{r}-\left(Y(t)+x_{2} c^{N(t)}\right)^{r}\right) c^{\beta N(t)} d t= \\
r \int_{0}^{\infty} \int_{x_{2}}^{x_{1}}\left(Y(t)+u c^{N(t}\right)^{r-1} c^{(\beta+1) N(t)} d u d t= \\
\quad r \int_{x_{2}}^{x_{1}} \int_{0}^{\infty}\left(Y(t)+u c^{N(t}\right)^{r-1} c^{(\beta+1) N(t)} d t d u .
\end{gathered}
$$

is an entire function in $r$ as long as $\operatorname{Re}(\beta)>-1$.

\section{Quantitative Results for Expected values of Inte- grals}

Let $\tau_{k}(k \geq 1)$ be the $k$-th event of the Poisson process after time zero. Even though (with probability one) time zero is not an event of the Poisson process, we let $\tau_{0}=0$, and $E_{k}=\tau_{k+1}-\tau_{k}$, so that $\left(E_{k}\right)_{k=0}^{\infty}$ are i.i.d., exponentially distributed with parameter $\lambda$. Now, as long as $r \neq-1$ :

$$
\begin{gathered}
\int_{0}^{\infty}\left(Y(t)+x c^{N(t)}\right)^{r} c^{\beta N(t)} d t=\sum_{k=0}^{\infty} \int_{\tau_{k}}^{\tau_{k+1}}\left(Y(t)+x c^{k}\right)^{r} c^{\beta k} d t= \\
\sum_{k=0}^{\infty} \frac{c^{\beta k}}{r+1}\left(\left(Y\left(\tau_{k}^{+}\right)+\left(\tau_{k+1}-\tau_{k}\right)+x c^{k}\right)^{r+1}-\left(Y\left(\tau_{k}^{+}\right)+x c^{k}\right)^{r+1}\right)= \\
\sum_{k=0}^{\infty} \frac{c^{\beta k}}{r+1}\left(Y\left(\tau_{k+1}^{-}\right)+x c^{k}\right)^{r+1}-\frac{x^{r+1}}{r+1}-\sum_{k=1}^{\infty} \frac{c^{\beta k}}{r+1}\left(c Y\left(\tau_{k}^{-}\right)+x c^{k}\right)^{r+1}=
\end{gathered}
$$




$$
-\frac{x^{r+1}}{r+1}+\frac{1-c^{r+\beta+1}}{r+1} \sum_{k=0}^{\infty} c^{\beta k}\left(Y\left(\tau_{k+1}^{-}\right)+x c^{k}\right)^{r+1} .
$$

It is easily seen that as long as $\operatorname{Re}(\beta)>0$ the re-arranging of terms above is warranted.

Next, we take expected values in (5.1). Clearly,

$$
Y\left(\tau_{k+1}^{-}\right)=\sum_{j=0}^{k} c^{j} E_{k-j}
$$

so that $Y\left(\tau_{k+1}^{-}\right)$has Laplace transform

$$
\prod_{j=0}^{k} \frac{1}{1+\frac{c^{j} s}{\lambda}}=\sum_{j=0}^{k} \frac{A_{j, k}}{1+\frac{c^{j} s}{\lambda}}
$$

and density

$$
\sum_{j=0}^{k} A_{j, k} \frac{\lambda}{c^{j}} e^{-\frac{\lambda}{c^{j}} y}
$$

where

$$
A_{j, k}=\prod_{\substack{\nu \neq j \\ 0 \leq \nu \leq k}} \frac{1}{1-c^{\nu-j}}=\frac{(-1)^{j} c^{\frac{1}{2} j(j+1)}}{(1-c) \cdots\left(1-c^{j}\right)(1-c) \cdots\left(1-c^{k-j}\right)}
$$

It is interesting to compare $A_{j, k}$ with $R_{j}$ in [2]. $A_{j, k}=R_{j} \prod_{\nu=1}^{\infty}\left(1-c^{k-j+\nu}\right)$ and $R_{j}=$ $\lim _{k \rightarrow \infty} A_{j, k}$. The random variable $Z$ in (1.5) has density

$$
\sum_{j=0}^{\infty} R_{j} \frac{\lambda}{c^{j}} e^{-\frac{\lambda}{c^{j}} z}
$$

We observe that by (A.4)

$$
\sum_{j=0}^{\infty}\left|A_{j, j+\nu}\right| c^{j(r+1+\beta)}=\frac{1}{(1-c) \cdots\left(1-c^{\nu}\right)} \prod_{\mu=0}^{\infty}\left(1+c^{r+2+\beta+\mu}\right)
$$

which on occasion can be used to prove absolute convergence, and we observe that

$$
\begin{aligned}
\sum_{j=0}^{k} A_{j, k} & =1, \sum_{j=0}^{\infty} R_{j}=1, \\
\sum_{j=0}^{\infty} A_{j, j+k} z^{j} & =\frac{\prod_{\nu=1}^{\infty}\left(1-c^{\nu} z\right)}{(1-c) \cdots\left(1-c^{k}\right)},
\end{aligned}
$$




$$
\begin{gathered}
-\left(1-c^{\beta}\right) \sum_{k=0}^{\infty} c^{\beta k} \sum_{j=0}^{k} j A_{j, k}= \\
\left(\prod_{\nu=0}^{\infty} \frac{1}{1-c^{\beta+1+\nu}}\right)\left(\sum_{j=1}^{\infty} \frac{(-1)^{j-1} j c^{\frac{1}{2} j(j+1)} c^{\beta j}}{(1-c) \cdots\left(1-c^{j}\right)}\right)= \\
\left(\sum_{\nu=0}^{\infty} \frac{(-1)^{\nu} c^{\frac{1}{2} \nu(\nu+1)} c^{\beta \nu}}{(1-c) \cdots\left(1-c^{\nu}\right)}\right)^{-1}\left(\sum_{j=1}^{\infty} \frac{(-1)^{j-1} j c^{\frac{1}{2} j(j+1)} c^{\beta j}}{(1-c) \cdots\left(1-c^{j}\right)}\right)= \\
\sum_{j=1}^{\infty} \frac{c^{\beta+j}}{1-c^{\beta+j}} .
\end{gathered}
$$

(5.10) uses (A.14). In (5.10) the last but one expression is the one to be used for numerical purposes, but in formulas we will use the more compact last expression.

Using the results above and the fact (see [43]) that

$$
\int_{0}^{\infty} e^{-y} \log (y) d y=\Gamma^{\prime}(1)=-\gamma=-.57721566490 \cdots
$$

where $\gamma$ is Euler's constant, we straightforwardly obtain that

$$
E[\log (Z)]=-\gamma-\log (\lambda)-(\log (c)) \sum_{j=1}^{\infty} \frac{c^{j}}{1-c^{j}}
$$

By (5.1)-(5.3)

$$
\begin{gathered}
E\left[\int_{0}^{\infty}\left(Y(t)+x c^{N(t)}\right)^{r} c^{\beta N(t)} d t\right]= \\
-\frac{x^{r+1}}{r+1}+\frac{1-c^{r+\beta+1}}{r+1} \sum_{k=0}^{\infty} c^{\beta k} \sum_{j=0}^{k} A_{j, k} \int_{0}^{\infty}\left(y+x c^{k}\right)^{r+1} \frac{\lambda}{c^{j}} e^{-\frac{\lambda}{c^{j}} y} d y= \\
-\frac{x^{r+1}}{r+1}+\frac{1-c^{r+\beta+1}}{(r+1) \lambda^{r+1}} \sum_{k=0}^{\infty} c^{\beta k} \sum_{j=0}^{k} A_{j, k} c^{j(r+1)} \int_{0}^{\infty}\left(v+\lambda x c^{k-j}\right)^{r+1} e^{-v} d v= \\
-\frac{x^{r+1}}{r+1}+\frac{1-c^{r+\beta+1}}{(r+1) \lambda^{r+1}} \sum_{k=0}^{\infty} c^{\beta k} \sum_{j=0}^{k} A_{j, k} c^{j(r+1)} e^{+\lambda x c^{k-j}} \int_{\lambda x c^{k-j}}^{\infty} u^{r+1} e^{-u} d u
\end{gathered}
$$


We can rewrite (5.13) with either the lower incomplete gamma functions $\gamma(a, x)$ or with the upper incomplete Gamma functions $\Gamma(a, x)$, see e.g. [43]. Since possibly $\operatorname{Re}(r)<-2$ we use the upper incomplete Gamma functions and get

$$
\begin{gathered}
E\left[\int_{0}^{\infty}\left(Y(t)+x c^{N(t)}\right)^{r} c^{\beta N(t)} d t\right]= \\
-\frac{x^{r+1}}{r+1}+\frac{1-c^{r+\beta+1}}{(r+1) \lambda^{r+1}} \sum_{\nu=0}^{\infty} c^{\beta \nu} \int_{0}^{\infty}\left(\left(v+\lambda x c^{\nu}\right)^{r+1} e^{-v} d v\right) \sum_{j=0}^{\infty} A_{j, j+\nu} c^{j(r+1+\beta)}= \\
-\frac{x^{r+1}}{r+1}+\frac{1}{(r+1) \lambda^{r+1}}\left(\prod_{\mu=0}^{\infty}\left(1-c^{r+1+\beta+\mu}\right)\right) \sum_{\nu=0}^{\infty} \frac{c^{\beta \nu} \int_{0}^{\infty}\left(v+\lambda x c^{\nu}\right)^{r+1} e^{-v} d v}{(1-c) \cdots\left(1-c^{\nu}\right)}= \\
-\frac{x^{r+1}}{r+1}+\frac{1}{(r+1) \lambda^{r+1}}\left(\prod_{\mu=0}^{\infty}\left(1-c^{r+1+\beta+\mu}\right)\right) \sum_{\nu=0}^{\infty} \frac{c^{\beta \nu} e^{\lambda x c^{\nu}} \Gamma\left(r+2, \lambda x c^{\nu}\right)}{(1-c) \cdots\left(1-c^{\nu}\right)} .
\end{gathered}
$$

The last but one step uses (A.13). To check whether the change in order of summation in (5.14) is warranted we use (5.7) and observe that (unless $(x=0$ and $\operatorname{Re}(r) \leq-1)$ ) the infinite sum in the RHS of (5.14) is finite when $\operatorname{Re}(r+\beta)>-2$, infinite when $r$ and $\beta$ are real and $r+\beta \leq-2$. The latter follows from the fact (easily verified by partial integration) that for $\lambda x>0,0<c<1$ and $a$ real:

$$
\lim _{\nu \rightarrow \infty} c^{-a \nu} \Gamma\left(a, \lambda x c^{\nu}\right)= \begin{cases}+\infty & \text { if } a \geq 0 \\ \frac{1}{|a|(\lambda x)^{|a|}} & \text { if } a<0\end{cases}
$$

Combining this with (5.7) we see that re-ordering of terms in the sums in (5.14) is warranted as long as $\operatorname{Re}(r+\beta)>-2$. Therefore (5.14) holds as long as $(\operatorname{Re}(\beta)>0$ and $\operatorname{Re}(r+\beta)>-2)$. The last expression in (5.14) will be useful in the next section.

If not only $\operatorname{Re}(\beta)>0$ and $\operatorname{Re}(r+\beta)>-2$ but also $\operatorname{Re}(r)>-2$ we can use the fact that $\Gamma\left(r+2, \lambda x c^{\nu}\right)=\Gamma(r+2)-\gamma\left(r+2, \lambda x c^{\nu}\right)$ to obtain (also using Lemma A.4 and other results from Appendix A):

$$
E\left[\int_{0}^{\infty}\left(Y(t)+x c^{N(t)}\right)^{r} c^{\beta N(t)} d t\right]=
$$




$$
\begin{gathered}
\frac{\Gamma(r+1)}{\lambda^{r+1}} \sum_{n=0}^{\infty} \frac{(\lambda x)^{n}}{n !}\left(\prod_{\mu=0}^{\infty} \frac{1-c^{r+1+\beta+\mu}}{1-c^{n+\beta+\mu}}\right) \\
-\frac{1}{\lambda^{r+1}} \sum_{n=1}^{\infty} \frac{(\lambda x)^{r+n}}{(r+1) \cdots(r+n)}\left(\prod_{\mu=1}^{n-1}\left(1-c^{r+\beta+\mu}\right)\right) .
\end{gathered}
$$

It must be noted that in the RHS of (5.16) both components have singularities in $r=-1,-2, \cdots$. These singularities annihilate each other: the LHS in (5.16) is an entire function of $r$ (as long as $\operatorname{Re}(\beta)>0$ and $x>0$ ). Thus, while the proof of (5.16) depends on $\operatorname{Re}(r)>-2$, the result, by analytic continuation, is valid for all $r$ and can be used to compute the expected value of the integral as long as $\operatorname{Re}(\beta)>0$ and $r$ is not a negative integer. As function of $\beta$, the first component in the RHS has singularities (simple poles) in $\beta=0,-1,-2, \cdots$.

If $r$ is a non-negative integer, $r=k,(5.16)$ becomes

$$
\begin{gathered}
E\left[\int_{0}^{\infty}\left(Y(t)+x c^{N(t)}\right)^{k} c^{\beta N(t)} d t\right]= \\
\frac{k !}{\lambda^{k+1}} \sum_{\nu=0}^{k} \frac{(\lambda x)^{\nu}}{\nu !} \frac{1}{\left(1-c^{\nu+\beta}\right) \cdots\left(1-c^{k+\beta}\right)},
\end{gathered}
$$

from which we obtain an alternative derivation of (3.21).

From (5.16) we easily see that for every $k \in\{0,1,2, \cdots\}, \quad r \notin\{-k,-k+1, \cdots,-1\}$,

$$
\begin{gathered}
E\left[\int_{0}^{\infty}\left(Y(t)+x c^{N(t)}\right)^{r} c^{\beta N(t)} d t\right]= \\
-\frac{1}{\lambda^{r+1}} \sum_{j=1}^{k} \frac{(\lambda x)^{r+j}}{r+j}\left(\prod_{\mu=1}^{j-1} \frac{1-c^{r+\beta+\mu}}{r+\mu}\right)+ \\
\lambda^{k}\left(\prod_{\mu=1}^{k} \frac{1-c^{r+\beta+\mu}}{r+\mu}\right) E\left[\int_{0}^{\infty}\left(Y(t)+x c^{N(t)}\right)^{r+k} c^{\beta N(t)} d t\right] .
\end{gathered}
$$

An alternate way to prove (5.18) is directly from (5.14), using the observation that $e^{x} \Gamma(r, x)=-\frac{x^{r}}{r}+\frac{x^{r}}{r} \Gamma(r+1, x)$. 
By continuity, (5.18) shows that for $k \in\{2,3, \cdots\}$

$$
\begin{gathered}
E\left[\int_{0}^{\infty}\left(Y(t)+x c^{N(t)}\right)^{-k} c^{\beta N(t)} d t\right]= \\
-\sum_{j=1}^{k-1} \lambda^{j-1} \frac{x^{-k+j}}{-k+j}\left(\prod_{\mu=1}^{j-1} \frac{1-c^{-k+\beta+\mu}}{-k+\mu}\right) \\
+\lambda^{k-1}\left(\prod_{\mu=1}^{k-1} \frac{1-c^{-k+\beta+\mu}}{-k+\mu}\right) E\left[\int_{0}^{\infty}\left(Y(t)+x c^{N(t)}\right)^{-1} c^{\beta N(t)} d t\right] .
\end{gathered}
$$

We will now study the case $r=-1$. If $r=-1$ we obtain, following the same recipe as in (5.1) etc:

$$
\begin{gathered}
\int_{0}^{\infty} \frac{c^{\beta N(t)}}{Y(t)+x c^{N(t)}} d t= \\
-\log (x)-\frac{c^{\beta} \log (c)}{1-c^{\beta}}+\left(1-c^{\beta}\right) \sum_{k=0}^{\infty} c^{\beta k} \log \left(Y\left(\tau_{k}^{-}\right)+x c^{k}\right)
\end{gathered}
$$

hence

$$
\begin{gathered}
E\left[\int_{0}^{\infty} \frac{c^{\beta N(t)}}{Y(t)+x c^{N(t)}} d t\right]= \\
-\log (x)-\frac{c^{\beta} \log (c)}{1-c^{\beta}}+\left(1-c^{\beta}\right) \sum_{k=0}^{\infty} c^{\beta k} E\left[\log \left(Y\left(\tau_{k}^{-}\right)+x c^{k}\right)\right] .
\end{gathered}
$$

Since ordinary convergence implies convergence in the sense of Abel, we have

$$
\lim _{\beta \downarrow 0}\left(1-c^{\beta}\right) \sum_{k=0}^{\infty} c^{\beta k} E\left[\log \left(Y\left(\tau_{k}^{-}\right)+x c^{k}\right)\right]=E[\log (Z)]
$$

independent of $x \geq 0$, where of course $Z$ has the distribution as in (1.5). For many purposes there is no need to work out (5.21) in more detail. In case more detail is needed, we easily obtain (using ideas similar to Lemma A.4 and the proof of (5.16))

$$
\begin{gathered}
E\left[\int_{0}^{\infty} \frac{c^{\beta N(t)}}{Y(t)+x c^{N(t)}} d t\right]= \\
-\log (\lambda x)-\log (c)\left(\sum_{j=0}^{\infty} \frac{c^{\beta+j}}{1-c^{\beta+j}}\right)
\end{gathered}
$$




$$
\begin{gathered}
+\left(\prod_{j=0}^{\infty}\left(1-c^{\beta+j}\right)\right) \sum_{\nu=0}^{\infty} \frac{c^{\beta \nu}}{(1-c) \cdots\left(1-c^{\nu}\right)} \int_{0}^{\infty}\left(\log \left(v+\lambda x c^{\nu}\right)\right) e^{-v} d v= \\
-\log (\lambda x)-\gamma \sum_{\nu=0}^{\infty} \frac{(\lambda x)^{\nu}}{\nu !}\left(1-c^{\beta}\right) \cdots\left(1-c^{\beta+\nu-1}\right)-\log (c)\left(\sum_{j=0}^{\infty} \frac{c^{\beta+j}}{1-c^{\beta+j}}\right) \\
-\left(\prod_{j=0}^{\infty}\left(1-c^{\beta+j}\right)\right) \sum_{\nu=0}^{\infty} \frac{c^{\beta \nu} e^{\lambda x c^{\nu}} \int_{0}^{\lambda x c^{\nu}} \log (u) e^{-u} d u}{(1-c) \cdots\left(1-c^{\nu}\right)} .
\end{gathered}
$$

We can rewrite further using Lemma A.5. Such a re-write shows that

$$
\sum_{\nu=0}^{\infty} \frac{c^{\beta \nu} e^{\lambda x c^{\nu}} \int_{0}^{\lambda x c^{\nu}} \log (u) e^{-u} d u}{(1-c) \cdots\left(1-c^{\nu}\right)}
$$

as function of $\beta$ is analytic on $\operatorname{Re}(\beta)>-1$ and has a pole of order 2 in $\beta=-1$, so that

$$
\left(\prod_{j=0}^{\infty}\left(1-c^{\beta+j}\right)\right) \sum_{\nu=0}^{\infty} \frac{c^{\beta \nu} e^{\lambda x c^{\nu}} \int_{0}^{\lambda x c^{\nu}} \log (u) e^{-u} d u}{(1-c) \cdots\left(1-c^{\nu}\right)}
$$

as function of $\beta$ is analytic on $\operatorname{Re}(\beta)>-1$, has a pole of order 1 in $\beta=-1$, and for $\beta=0$ is zero for all values of $x$.

We can substitute (5.23) into (5.19) to take care of the situation $r \in\{-2,-3, \cdots\}$.

If the initial value $X(0)$ is random, we can take expected values in (5.14), (5.16), (5.18), (5.19), (5.23).

The most interesting such situation is where $X(0)$ has the distribution of $Z$ in (1.5), so that all $X(t)$ has that same distribution. However, in that situation, simply replacing $x^{\nu}$ by $E\left[Z^{\nu}\right]$ and using (1.6) does not work because in (5.16) it leads to $\infty-\infty$. Instead we use the fact that (for $\operatorname{Re}(r)>-2$, and with the substitution $x=v+\lambda z c^{\nu}, \quad y=v-\lambda z c^{\nu}$ )

$$
\begin{array}{r}
E\left[\int_{0}^{\infty}\left(v+\lambda Z c^{\nu}\right)^{r+1} e^{-v} d v\right]=\sum_{j=0}^{\infty} R_{j} \frac{\lambda}{c^{j}} \int_{0}^{\infty} \int_{0}^{\infty}\left(v+\lambda z c^{\nu}\right)^{r+1} e^{-v} e^{-\frac{\lambda}{c^{j}} z} d v d z= \\
\sum_{j=0}^{\infty} \frac{R_{j}}{2 c^{\nu+j}} \int_{0}^{\infty} x^{r+1} e^{-\frac{x}{2}\left(\frac{1}{c^{\nu+j}}+1\right)} \int_{-x}^{+x} e^{\frac{y}{2}\left(\frac{1}{c^{\nu+j}}-1\right)} d y d x=\Gamma(r+2) \sum_{j=0}^{\infty} R_{j} \frac{1-c^{(r+2)(\nu+j)}}{1-c^{\nu+j}}
\end{array}
$$


where if $\nu=j=0 \quad \frac{1-c^{(r+2)(\nu+j)}}{1-c^{\nu+j}}$ is interpreted as $(r+2)$.

For the case $r=-1$ we similarly obtain

$$
E\left[\int_{0}^{\infty}\left(\log \left(v+\lambda Z c^{\nu}\right)\right) e^{-v} d v\right]=-\gamma-(\log (c)) \sum_{j=0}^{\infty} R_{j} \frac{(\nu+j) c^{\nu+j}}{1-c^{\nu+j}}
$$

where if $\nu=j=0 \quad \frac{\nu+j}{1-c^{\nu+j}}$ is interpreted as $\frac{-1}{\log (c)}$.

(5.26), (5.27) can be substituted into (5.14), (5.18), (5.19), (5.23). For $\operatorname{Re}(\beta)>$ $0, \operatorname{Re}(r+\beta)>-2, r \neq-1$ this yields

$$
\begin{gathered}
E\left[\int_{0}^{\infty}\left(Y(t)+Z c^{N(t)}\right)^{r} c^{\beta N(t)} d t\right]= \\
-\frac{E\left[Z^{r+1}\right]}{r+1}+\frac{1}{(r+1) \lambda^{r+1}}\left(\prod_{\mu=0}^{\infty}\left(1-c^{r+1+\beta+\mu}\right)\right) \sum_{\nu=0}^{\infty} \frac{c^{\beta \nu} E\left[\int_{0}^{\infty}\left(v+\lambda Z c^{\nu}\right)^{r+1} e^{-v} d v\right]}{(1-c) \cdots\left(1-c^{\nu}\right)}= \\
-\frac{\Gamma(r+1)}{\lambda^{r+1}}\left(\prod_{k=1}^{\infty} \frac{1-c^{r+1+k}}{1-c^{k}}\right) \\
+\frac{\Gamma(r+1)}{\lambda^{r+1}}\left(\prod_{k=1}^{\infty} \frac{1-c^{r+\beta+k}}{1-c^{k}}\right) \sum_{\mu=0}^{\infty} c^{\beta \mu} \frac{1-c^{(r+2) \mu}}{1-c^{\mu}} \frac{\left(1-c^{1-\beta}\right) \cdots\left(1-c^{\mu-\beta}\right)}{(1-c) \cdots\left(1-c^{\mu}\right)},
\end{gathered}
$$

where if $\mu=0 \quad \frac{1-c^{(r+2) \mu}}{1-c^{\mu}}$ is interpreted as $(r+2)$.

The first component in the RHS of (5.28) is a function of $r$ only and has only one singularity (a simple pole in $r=-1$ ). The other singularities of $\Gamma(r+1)$ are annihilated by the infinite product. The infinite sum in the second component of the RHS of (5.28) converges for $\operatorname{Re}(\beta)>0, \operatorname{Re}(r+\beta)>-2$, We have not succeeded in sufficiently simplifying that infinite sum in the second component of the RHS of (5.28). Because of the first remark following (5.28) the second component has, for $\operatorname{Re}(\beta>0$, as function of $r$, only one singularity, namely a simple pole in $r=-1$.

Similarly, (5.27) yields

$$
E\left[\int_{0}^{\infty} \frac{c^{\beta N(t)}}{\left(Y(t)+Z c^{N(t)}\right)} d t\right]=
$$




$$
\begin{gathered}
-E[\log (\lambda Z)]-(\log (c)) \sum_{j=0}^{\infty} \frac{c^{\beta+j}}{1-c^{\beta+j}}+\left(\prod_{j=0}^{\infty}\left(1-c^{\beta+j}\right)\right) \sum_{\nu=0}^{\infty} \frac{c^{\beta \nu} E\left[\int_{0}^{\infty}\left(\log \left(v+\lambda Z c^{\nu}\right)\right) e^{-v} d v\right]}{(1-c) \cdots\left(1-c^{\nu}\right)}= \\
(\log (c))\left(\left(\sum_{j=1}^{\infty} \frac{c^{j}}{1-c^{j}}\right)-\left(\sum_{j=0}^{\infty} \frac{c^{\beta+j}}{1-c^{\beta+j}}\right)\right) \\
-(\log (c))\left(\prod_{j=0}^{\infty} \frac{1-c^{\beta+j}}{1-c^{j+1}}\right) \sum_{\mu=0}^{\infty} \frac{\mu c^{(1+\beta) \mu}}{1-c^{\mu}} \frac{\left(1-c^{1-\beta}\right) \cdots\left(1-c^{\mu-\beta}\right)}{(1-c) \cdots\left(1-c^{\mu}\right)}
\end{gathered}
$$

where if $\mu=0 \quad \frac{\mu}{1-c^{\mu}}$ is interpreted as $\frac{-1}{\log (c)}$. We have not succeeded in sufficiently simplifying the infinite sum in the second component of the RHS of (5.29). It converges for $\operatorname{Re}(\beta)>-1$. The first component converges for $\operatorname{Re}(\beta)>0$.

It may be of interest to observe that with $\beta=1$ the last expression reduces to

$$
E\left[\int_{0}^{\infty} \frac{c^{N(t)}}{Y(t)+Z c^{N(t)}} d t\right]=1
$$

We can use (5.18) to obtain that for any $n \in\{0,1, \cdots\}$

$$
\begin{gathered}
E\left[\int_{0}^{\infty}\left(Y(t)+Z c^{N(t)}\right)^{r} c^{\beta N(t)} d t\right]= \\
-\sum_{j=1}^{n} \lambda^{j-1} \frac{E\left[Z^{r+j}\right]}{r+j}\left(\prod_{\mu=1}^{j-1} \frac{1-c^{r+\beta+\mu}}{r+\mu}\right) \\
+\lambda^{n}\left(\prod_{\mu=1}^{n} \frac{1-c^{r+\beta+\mu}}{r+\mu}\right) E\left[\int_{0}^{\infty}\left(Y(t)+Z c^{N(t)}\right)^{r+n} c^{\beta N(t)} d t\right] .
\end{gathered}
$$

For $r=-k \in\{-2,-3, \cdots\}$ we choose $n=k-1$ and use (5.29). Otherwise, we choose $n$ such that $\operatorname{Re}(r+n+\beta)>-2$. In that case it is an interesting problem to choose the "best" value of $n$.

It must be noted that while, in (5.28), (5.29), (5.30), (5.31), $X(t)=\left(Y(t)+Z c^{N(t)}\right)$ always has the stationary distribution, i.e. the distribution of $Z$, no such simple result holds for $X(t)^{r} c^{\beta N(t)}=\left(Y(t)+Z c^{N(t)}\right)^{r} c^{\beta N(t)}$. 
Because "ordinary" convergence implies convergence in the sense of Abel, (5.15) also shows (for convenience of the reader we changed the sign of $r$ ) that if $\operatorname{Re}(r)>2$ then

$$
\begin{gathered}
E\left[\int_{0}^{\infty} \frac{1}{\left(Y(t)+x c^{N(t)}\right)^{r}} c^{(r-2) N(t)} d t\right]= \\
\lim _{\beta \downarrow r-2}\left[\int_{0}^{\infty} \frac{1}{\left(Y(t)+x c^{N(t)}\right)^{r}} c^{\beta N(t)} d t\right]= \\
\frac{1}{(r-1) x^{r-1}}+\frac{(1-c) \lambda}{c(r-1)(r-2) x^{r-2}} .
\end{gathered}
$$

Another case where (5.14) gives a simple answer is the case " $r+\beta=-1$ ". For ease of reading we again change the sign of $r$ and get: If $\operatorname{Re}(r)>1$ then

$$
E\left[\int_{0}^{\infty} \frac{1}{\left(Y(t)+x c^{N(t)}\right)^{r}} c^{(r-1) N(t)} d t\right]=\frac{1}{(r-1) x^{r-1}} .
$$

\section{Expected values of integrals of differences}

In this section we obtain expressions for

$$
D\left(x_{1}, x_{2}, r, \beta\right)=E\left[\int_{0}^{\infty}\left(\left(Y(t)+x_{1} c^{N(t)}\right)^{r}-\left(Y(t)+x_{2} c^{N(t)}\right)^{r}\right) c^{\beta N(t)} d t\right],
$$

and

$$
D(x, Z, r, \beta)=E\left[\int_{0}^{\infty}\left(\left(Y(t)+x c^{N(t)}\right)^{r}-\left(Y(t)+Z c^{N(t)}\right)^{r}\right) c^{\beta N(t)} d t\right],
$$

i.e., we evaluate integrals of differences as in (5.1) and their expected values, and also similar differences between starting with $X(0)=x$ and starting with $X(0)=Z$.

From (5.16) we immediately obtain

$$
\begin{gathered}
D\left(x_{1}, x_{2}, r, \beta\right)= \\
\frac{\Gamma(r+1)}{\lambda^{r+1}}\left(\prod_{\mu=1}^{\infty} \frac{1-c^{r+\beta+\mu}}{1-c^{\beta+\mu}}\right) \sum_{n=1}^{\infty} \frac{\lambda^{n}}{n !}\left(x_{1}^{n}-x_{2}^{n}\right)\left(\prod_{\mu=1}^{n-1}\left(1-c^{\beta+\mu}\right)\right)
\end{gathered}
$$




$$
-\frac{1}{\lambda^{r+1}} \sum_{n=1}^{\infty} \frac{\lambda^{r+n}\left(x_{1}^{r+n}-x_{2}^{r+n}\right)}{(r+1) \cdots(r+n)}\left(\prod_{\mu=1}^{n-1}\left(1-c^{r+\beta+\mu}\right)\right) .
$$

In (6.3), if $r+n=0$ then $\frac{\left(x_{1}^{r+n}-x_{2}^{r+n}\right)}{r+n}$ is interpreted as $\log \left(\frac{x_{1}}{x_{2}}\right)$. Both components in the RHS still have singularities in $r=-1,-2, \cdots$. These singularities still annihilate each other. As function of $\beta$, the first component in the RHS of (6.3) has singularities (simple poles) in $\beta=-1,-2, \cdots$.

For $r=-1$ we obtain from $(5.23)$

$$
\begin{gathered}
D\left(x_{1}, x_{2},-1, \beta\right)= \\
-\log \left(\frac{x_{1}}{x_{2}}\right)-\gamma \sum_{\nu=1}^{\infty} \frac{\lambda^{\nu}\left(x_{1}^{\nu}-x_{2}^{\nu}\right)}{\nu !}\left(1-c^{\beta}\right) \cdots\left(1-c^{\beta+\nu-1}\right) \\
-\left(\prod_{j=0}^{\infty}\left(1-c^{\beta+j}\right)\right) \sum_{\nu=0}^{\infty} \frac{c^{\beta \nu}\left(e^{\lambda x_{1} c^{\nu}} \int_{0}^{\lambda x_{1} c^{\nu}}(\log (u)) e^{-u} d u-e^{\lambda x_{2} c^{\nu}} \int_{0}^{\lambda x_{2} c^{\nu}}(\log (u)) e^{-u} d u\right)}{(1-c) \cdots\left(1-c^{\nu}\right)} .
\end{gathered}
$$

For $r \in\{-2,-3, \cdots\}$ we use (5.19) and (6.4). We leave this result as an exercise for the reader.

In order to prepare for $(1.16)$ we obtain, for $\operatorname{Re}(r)>-2, \quad r \neq-1, \operatorname{Re}(\beta)>-1$

$$
\begin{gathered}
E\left[\int_{0}^{\infty}\left(\left(Y(t)-x c^{N(t)}\right)^{r}-\left(Y(t)-Z c^{N(t)}\right)^{r}\right) c^{\beta N(t)} d t\right]= \\
-\frac{x^{r+1}-E\left[Z^{r+1}\right]}{r+1} \\
+\frac{1}{(r+1) \lambda^{r+1}}\left(\prod_{\mu=0}^{\infty}\left(1-c^{r+1+\beta+\mu}\right)\right) \times \\
\sum_{\nu=0}^{\infty} \frac{c^{\beta \nu}}{(1-c) \cdots\left(1-c^{\nu)}\right.}\left(\int_{0}^{\infty}\left(v+\lambda x c^{\nu}\right)^{r+1} e^{-v} d v-\Gamma(r+2) \sum_{j=0}^{\infty} R_{j} \frac{1-c^{(r+2)(\nu+j)}}{1-c^{\nu+j}}\right) .
\end{gathered}
$$

Now:

$$
\int_{0}^{\infty}\left(v+\lambda x c^{\nu}\right)^{r+1} e^{-v} d v-\Gamma(r+2) \sum_{j=0}^{\infty} R_{j} \frac{1-c^{(r+2)(\nu+j)}}{1-c^{\nu+j}}=
$$




$$
\begin{gathered}
e^{\lambda x c^{\nu}} \Gamma(r+2)-e^{\lambda x c^{\nu}} \gamma\left(r+2, \lambda x c^{\nu}\right)-\Gamma(r+2) \sum_{j=0}^{\infty} R_{j} \frac{1-c^{(r+2)(\nu+j)}}{1-c^{\nu+j}}= \\
\left(e^{\lambda x c^{\nu}}-1\right) \Gamma(r+2)-e^{\lambda x c^{\nu}} \gamma\left(r+2, \lambda x c^{\nu}\right)+\Gamma(r+2)\left(1-\sum_{j=0}^{\infty} R_{j} \frac{1-c^{(r+2)(\nu+j)}}{1-c^{\nu+j}}\right)= \\
\Gamma(r+2)\left(e^{\lambda x c^{\nu}}-1\right)-e^{\lambda x c^{\nu}} \gamma\left(r+2, \lambda x c^{\nu}\right)-\Gamma(r+2) \sum_{j=0}^{\infty} R_{j} c^{\nu+j} \frac{1-c^{(r+1)(\nu+j)}}{1-c^{\nu+j}},
\end{gathered}
$$

which after some (by now standard) arithmetic yields

$$
\begin{gathered}
E\left[\int_{0}^{\infty}\left(\left(Y(t)-x c^{N(t)}\right)^{r}-\left(Y(t)-Z c^{N(t)}\right)^{r}\right) c^{\beta N(t)} d t\right]= \\
\frac{\Gamma(r+1)}{\lambda^{r+1}} \sum_{n=1}^{\infty} \frac{(\lambda x)^{n}}{n !}\left(\prod_{\mu=0}^{\infty} \frac{1-c^{r+1+\beta+\mu}}{1-c^{n+\beta+\mu}}\right) \\
-\frac{1)}{\lambda^{r+1}} \sum_{n=1}^{\infty} \frac{(\lambda x)^{r+n}}{(r+1) \cdots(r+n)}\left(1-c^{r+1+\beta}\right) \cdots\left(1-c^{r+n-1+\beta}\right) \\
+\frac{\Gamma(r+1)}{\lambda^{r+1}}\left(\prod_{\mu=1}^{\infty} \frac{1-c^{r+1+\mu}}{1-c^{\mu}}\right) \\
-\frac{\Gamma(r+1)}{\lambda^{r+1}}\left(\prod_{\mu=1}^{\infty} \frac{1-c^{r+\beta+\mu}}{1-c^{\mu}}\right) \sum_{n=0}^{\infty} c^{(1+\beta) n} \frac{1-c^{(r+1) n}}{1-c^{n}} \frac{\left(1-c^{1-\beta}\right) \cdots\left(1-c^{n-\beta}\right)}{(1-c) \cdots\left(1-c^{n}\right)}
\end{gathered}
$$

where if $n=0 \quad \frac{1-c^{(r+1) n}}{1-c^{n}}$ is interpreted as $r+1$. While the derivation uses the fact that $\operatorname{Re}(r)>-2$, we see by analytic continuation that the result holds for $\operatorname{Re}(\beta)>$ $-1, \quad \operatorname{Re}(r+\beta)>-2, \quad r \notin\{-1,-2, \cdots\}$.

For $r=-1$ we combine (5.23) and (5.29) and obtain

$$
\begin{gathered}
E\left[\int_{0}^{\infty}\left(\frac{1}{Y(t)+x c^{N(t)}}-\frac{1}{Y(t)+Z c^{N(t)}}\right) c^{\beta N(t)} d t\right]= \\
-\log (\lambda x)-\gamma \sum_{\nu=0}^{\infty} \frac{(\lambda x)^{\nu}}{\nu !}\left(1-c^{\beta}\right) \cdots\left(1-c^{\beta+\nu-1}\right)-(\log (c)) \sum_{j=1}^{\infty} \frac{c^{j}}{1-c^{j}} \\
-\left(\prod_{j=0}^{\infty}\left(1-c^{\beta+j}\right)\right) \sum_{\nu=0}^{\infty} \frac{c^{\beta \nu} e^{\lambda x c^{\nu}} \int_{0}^{\lambda x c^{\nu}} \log (u) e^{-u} d u}{(1-c) \cdots\left(1-c^{\nu}\right)}
\end{gathered}
$$




$$
-(\log (c))\left(\prod_{j=0}^{\infty} \frac{1-c^{\beta+j}}{1-c^{j+1}}\right) \sum_{\mu=0}^{\infty} \frac{\mu c^{(1+\beta) \mu}}{1-c^{\mu}} \frac{\left(1-c^{1-\beta}\right) \cdots\left(1-c^{\mu-\beta}\right)}{(1-c) \cdots\left(1-c^{\mu}\right)}
$$

where if $\mu=0 \frac{\mu}{1-c^{\mu}}$ still is interpreted as $\frac{-1}{\log (c)}$. (6.8) converges for $\operatorname{Re}(\beta)>-1$.

The case

$$
E\left[\int_{0}^{\infty}\left(\left(Y(t)+x c^{N(t)}\right)^{r}-\left(Y(t)+Z c^{N(t)}\right)^{r}\right) c^{\beta N(t)} d t\right]
$$

with $\operatorname{Re}(\beta)>-1, \quad \operatorname{Re}(r+\beta) \leq-2$ is left as an exercise for the reader.

Note that $(6.3),(6.4,(6.7)$ and (6.8) all four, as functions of $\beta$, are analytic on $\operatorname{Re}(\beta)>$ -1 , so that in the next section setting $\beta=0$ will not be a problem.

\section{$7 \quad$ The case $\beta=0$}

(6.3) with $\beta=0$ yields

$$
\begin{gathered}
D\left(x_{1}, x_{2}, r, 0\right)= \\
\frac{\Gamma(r+1)}{\lambda^{r+1}} \sum_{n=1}^{\infty} \frac{\lambda^{n}}{n !}\left(x_{1}^{n}-x_{2}^{n}\right)\left(\prod_{\mu=1}^{\infty} \frac{1-c^{r+\mu}}{1-c^{n-1+\mu}}\right)-\sum_{n=1}^{\infty} \lambda^{n-1} \frac{x_{1}^{r+n}-x_{2}^{r+n}}{r+n}\left(\prod_{\mu=1}^{n-1} \frac{1-c^{r+\mu}}{r+\mu}\right),
\end{gathered}
$$

where if $r+n=0 \quad \frac{x_{1}^{r+n}-x_{2}^{r+n}}{r+n}$ is interpreted as $\log \left(\frac{x_{1}}{x_{2}}\right)$, and if $r+\mu=0 \quad \frac{1-c^{r+\mu}}{r+\mu}$ is interpreted as $-\log (c)$.

If $r=-k \in\{-1,-2, \cdots\}$ then $(\Gamma(r+1))\left(1-c^{r+k}\right)$ is interpreted as $\frac{(-1)^{k} \log (c)}{(k-1) !}$.

If $r=k \in\{0,1,2, \cdots\}$ (7.1) reduces to

$$
\begin{gathered}
D\left(x_{1}, x_{2}, k, 0\right)= \\
\frac{k !}{\lambda^{k+1}} \sum_{\nu=1}^{k} \lambda^{\nu} \frac{x_{1}^{\nu}-x_{2}^{\nu}}{\nu !} \frac{1}{\left(1-c^{\nu}\right) \cdots\left(1-c^{k}\right)} .
\end{gathered}
$$

If $r=-1(7.1)$ reduces to

$$
D\left(x_{1}, x_{2},-1,0\right)=-\log \left(\frac{x_{1}}{x_{2}}\right) .
$$


The case $D\left(x_{1}, x_{2},-k, 0\right)$ with $k \in\{2,3, \cdots\}$ is left as an exercise for the reader.

From (6.7) we see that for $\operatorname{Re}(r)>-2, \quad r \neq-1$

$$
\begin{gathered}
D(x, Z, r, 0)= \\
\frac{\Gamma(r+1)}{\lambda^{r+1}} \sum_{n=1}^{\infty} \frac{(\lambda x)^{n}}{n !}\left(\prod_{\mu=0}^{\infty} \frac{1-c^{r+1+\mu}}{1-c^{n+\beta+\mu}}\right) \\
-\frac{1}{\lambda^{r+1}} \sum_{n=1}^{\infty} \frac{(\lambda x)^{r+n}}{(r+1) \cdots(r+n)}\left(1-c^{r+1}\right) \cdots\left(1-c^{r+n-1}\right) \\
+\frac{\Gamma(r+1)}{\lambda^{r+1}}\left(\prod_{\mu=1}^{\infty} \frac{1-c^{r+1+\mu}}{1-c^{\mu}}\right) \\
-\frac{\Gamma(r+1)}{\lambda^{r+1}}\left(\prod_{\mu=1}^{\infty} \frac{1-c^{r+\mu}}{1-c^{\mu}}\right) \sum_{n=0}^{\infty} c^{n} \frac{1-c^{(r+1) n}}{1-c^{n}},
\end{gathered}
$$

where if $n=0 \frac{1-c^{(r+1) n}}{1-c^{n}}$ is interpreted as $r+1$.

For $r=-1$ (6.8) gives

$$
\begin{gathered}
D(x, Z,-1,0)= \\
-\log (\lambda x)-\gamma-(\log (c)) \sum_{j=1}^{\infty} \frac{c^{j}}{1-c^{j}} .
\end{gathered}
$$

The case of $D(x, Z, r, 0)$ with $\operatorname{Re}(r) \leq-2$ we leave as an exercise to the reader.

\section{Transform Techniques}

We define:

$$
\psi(x, t, s, z)=E\left[\exp \left\{-s\left(Y(t)+x c^{N(t)}\right)\right\} z^{N(t)}\right] .
$$

Let y be the last point of the Poisson process $\left(\tau_{k}\right)_{k}$ in the interval $(0, t]$. With probability $e^{-\lambda t}$ there is no such point. This gives

$$
\psi(x, t, s, z)=e^{-\lambda t} e^{-s(x+t)}+\lambda z \int_{0}^{t} e^{-(s+\lambda)(t-y)} \psi(x, y, c s, z) d y .
$$


We define:

$$
\eta(x, \sigma, s, z)=\int_{0}^{\infty} e^{-\sigma t} \psi(x, t, s, z) d t
$$

With (8.2) this gives

$$
\eta(x, \sigma, s, z)=\frac{1}{\sigma+\lambda+s}\left(e^{-s x}+\lambda z \eta(x, \sigma, c s, z)\right) .
$$

Repeating this procedure we see that for all $k \in\{1,2, \cdots\}$

$$
\eta(x, \sigma, s, z)=\sum_{j=0}^{k-1} \frac{(\lambda z)^{j} \exp \left\{-s x c^{j}\right\}}{\prod_{\nu=0}^{j}\left(\sigma+\lambda+c^{\nu} s\right)}+\frac{(\lambda z)^{k}}{\prod_{\nu=0}^{k-1}\left(\sigma+\lambda+c^{\nu} s\right)} \eta\left(x, \sigma, c^{k} s, z\right) .
$$

For $|z| \leq 1, \operatorname{Re}(\sigma)>0, \operatorname{Re}(s)>0$ this gives

$$
\eta(x, \sigma, s, z)=\sum_{j=0}^{\infty} \frac{(\lambda z)^{j} \exp \left\{-s x c^{j}\right\}}{\prod_{\nu=0}^{j}\left(\sigma+\lambda+c^{\nu} s\right)} .
$$

For use in the the proof of Theorem 2 in the next section we re-write (8.6) as

$$
\eta(x, \sigma, s, z)=\sum_{k=0}^{\infty}\left(\frac{\lambda z}{\sigma+\lambda}\right)^{k} \frac{e^{-s x c^{k}}}{(\sigma+\lambda) \prod_{j=0}^{k}\left(1+\frac{s}{\sigma+\lambda} c^{j}\right)} .
$$

By partial fraction expansion we then prove that

$$
\begin{gathered}
\eta(x, \sigma, s, z)= \\
\frac{e^{-s x}}{\sigma+\lambda+s}+\sum_{k=1}^{\infty} z^{k} e^{-s x c^{k}} \sum_{j=0}^{k}(-1)^{j} c^{\frac{1}{2} j(j+1)}\left(\prod_{\nu=1}^{j} \frac{1}{1-c^{\nu}} \prod_{\nu=1}^{k-j} \frac{1}{1-c^{\nu}}\right)\left(\frac{\lambda}{\sigma+\lambda}\right)^{k} \frac{1}{\sigma+\lambda+s c^{j}}
\end{gathered}
$$

Hence

$$
\begin{gathered}
\psi(x, t, s, z)= \\
e^{-s x} e^{-t(\lambda+s)}+ \\
e^{-\lambda t} \sum_{k=1}^{\infty}(\lambda z)^{k} \sum_{j=0}^{k}(-1)^{j} c^{\frac{1}{2} j(j+1)}\left(\prod_{\nu=1}^{j} \frac{1}{1-c^{\nu}} \prod_{\nu=1}^{k-j} \frac{1}{1-c^{\nu}}\right) e^{-s x c^{k}} \int_{0}^{t} \frac{y^{k-1}}{(k-1) !} e^{-(t-y) s c^{j}} d y .
\end{gathered}
$$


In Section 9 we will formulate real domain results for transient densities which are a direct consequence of (8.9) above.

Before going to those transient densities we use (8.5) to give an alternative proof of (1.5). Using (3.3), (3.25) we obtain that for $|z| \leq 1, s \downarrow 0$,

$$
\begin{gathered}
\psi(x, t, 0, z)-\psi(x, t, s, z)=\exp \{-\lambda t(1-z)\}-\psi(x, t, s, z)= \\
s\left(\frac{\exp \{\lambda t(1-z)\}}{\lambda z(1-c)}+\left(x-\frac{1}{\lambda z(1-c)}\right) \exp \{-\lambda t(1-c z)\}\right)+o(s)(s \downarrow 0),
\end{gathered}
$$

where for all $0<x_{0}<\infty$ the $o(s)$ is uniform on $|z| \leq 1,0 \leq x \leq x_{0}, 0 \leq t<\infty$. Hence, for $k \in\{1,2, \cdots\}$ we have

$$
\begin{gathered}
\eta(x, \sigma, s, z)=\sum_{j=0}^{k-1} \frac{(\lambda z)^{j} \exp \left\{-s x c^{j}\right\}}{\prod_{\nu=0}^{j}\left(\sigma+\lambda+c^{\nu} s\right)}+ \\
\frac{(\lambda z)^{k}}{\prod_{\nu=0}^{k-1}\left(\sigma+\lambda+c^{\nu} s\right)}\left(\frac{1}{\sigma+\lambda(1-z)}-\frac{s c^{k}}{\sigma+\lambda(1-c z)}\left(x+\frac{1}{\sigma+\lambda(1-c)}\right)+\frac{o\left(s c^{k}\right)(k \uparrow \infty)}{\sigma}\right),
\end{gathered}
$$

where for every $0<s_{0}<\infty, 0<x_{0}<\infty$ the $o\left(s c^{k}\right)$ is uniform on $|z| \leq 1,0 \leq x \leq x_{0}, 0<$ $s \leq s_{0}, 0 \leq \sigma<\infty$. Hence:

$$
\psi(x, \infty, s, 1)=\lim _{\sigma \downarrow 0} \sigma \eta(x, \sigma, s, 1)=\left(\prod_{\nu=0}^{k-1} \frac{1}{1+s \frac{c^{\nu}}{\lambda}}\right)\left(1+o\left(s c^{k}\right)\right)(k \uparrow \infty) .
$$

We (again) obtain (1.5) by letting $k \uparrow \infty$.

\section{Transient Distributions}

The following lemma makes it easier to relate the various formulations of the main result in this section:

Lemma 5 For all $k \in\{1,2, \cdots\}$ and all $t$ and $u$

$$
\sum_{j=0}^{k}(-1)^{j} c^{\frac{1}{2} j(j+1)} c^{-k j}\left(\prod_{\nu=1}^{j} \frac{1}{1-c^{\nu}}\right)\left(\prod_{\nu=1}^{k-j} \frac{1}{1-c^{\nu}}\right)\left(t c^{j}+u\right)^{k-1}=0 .
$$


This lemma will be proven in Appendix A.

In the following, it always is understood that $x \geq 0$ and $t>0$. The main result in this section is

Theorem 2. For $k \geq 1$, choose any $y_{\min }, y_{\max }$ with

$$
-\infty \leq y_{\min } \leq(x+t) c^{k}<x c^{k}+t \leq y_{\max } \leq+\infty
$$

(where $y_{\min }, y_{\max }$ are allowed to depend on $k$ ). Then (for $k \geq 1$ ):

$$
\begin{gathered}
\frac{d}{d y} P\{N(t)=k, X(t) \leq y \mid X(0)=x\}= \\
\frac{e^{-\lambda t} \lambda^{k}}{(k-1) !} \sum_{j=0}^{k}(-1)^{j} c^{\frac{1}{2} j(j+1)} c^{-k j}\left(\prod_{\nu=1}^{j} \frac{1}{1-c^{\nu}}\right)\left(\prod_{\nu=1}^{k-j} \frac{1}{1-c^{\nu}}\right) \times \\
\left(x c^{k}+t c^{j}-y\right)^{k-1} \chi\left(y_{\min }<y<x c^{k}+t c^{j}\right)= \\
\frac{e^{-\lambda t} \lambda^{k}}{(k-1) !} \sum_{j=0}^{k}(-1)^{k-j} c^{\frac{1}{2} j(j+1)} c^{-k j}\left(\prod_{\nu=1}^{j} \frac{1}{1-c^{\nu}}\right)\left(\prod_{\nu=1}^{k-j} \frac{1}{1-c^{\nu}}\right) \times \\
\left(y-\left(x c^{k}+t c^{j}\right)\right)^{k-1} \chi\left(x c^{k}+t c^{j}<y<y_{\max }\right) .
\end{gathered}
$$

Because of (9.1) the actual values of $y_{\min }$ and $y_{\max }$ do not matter, as long as they satisfy $(9.2)$.

For $k=0$ :

$$
P\{N(t)=0, X(t) \leq y \mid X(0)=x\}= \begin{cases}0 & \text { if } y<x+t \\ e^{-\lambda t} & \text { if } y \geq x+t\end{cases}
$$

For $y_{\min }<y<y_{\max }$ and $k \geq 1$ choose $n=n(y, k)$ such that

$$
n= \begin{cases}k & \text { if } y \leq(x+t) c^{k} \\ \text { such that } x c^{k}+t c^{n+1}<y \leq x c^{k}+t c^{n} & \text { if }(x+t) c^{k}<y \leq x c^{k}+t \\ -1 & \text { if } x c^{k}+t<y .\end{cases}
$$


The two expressions in (9.3) are reconciled by observing that

$$
\begin{gathered}
\sum_{j=0}^{k}(-1)^{j} c^{\frac{1}{2} j(j+1)} c^{-k j}\left(\prod_{\nu=1}^{j} \frac{1}{1-c^{\nu}}\right)\left(\prod_{\nu=1}^{k-j} \frac{1}{1-c^{\nu}}\right) \times \\
\left(x c^{k}+t c^{j}-y\right)^{k-1} \chi\left(y_{\min }<y<x c^{k}+t c^{j}\right)= \\
\sum_{j=0}^{n}(-1)^{j} c^{\frac{1}{2} j(j+1)} c^{-k j}\left(\prod_{\nu=1}^{j} \frac{1}{1-c^{\nu}}\right)\left(\prod_{\nu=1}^{k-j} \frac{1}{1-c^{\nu}}\right)\left(x c^{k}+t c^{j}-y\right)^{k-1}= \\
-\sum_{j=n+1}^{k}(-1)^{j} c^{\frac{1}{2} j(j+1)} c^{-k j}\left(\prod_{\nu=1}^{j} \frac{1}{1-c^{\nu}}\right)\left(\prod_{\nu=1}^{k-j} \frac{1}{1-c^{\nu}}\right)\left(x c^{k}+t c^{j}-y\right)^{k-1}= \\
\sum_{j=n+1}^{k}(-1)^{k-j} c^{\frac{1}{2} j(j+1)} c^{-k j}\left(\prod_{\nu=1}^{j} \frac{1}{1-c^{\nu}}\right)\left(\prod_{\nu=1}^{k-j} \frac{1}{1-c^{\nu}}\right)\left(y-\left(x c^{k}+t c^{j}\right)\right)^{k-1}= \\
\sum_{j=0}^{k}(-1)^{k-j} c^{\frac{1}{2} j(j+1)} c^{-k j}\left(\prod_{\nu=1}^{j} \frac{1}{1-c^{\nu}}\right)\left(\prod_{\nu=1}^{k-j} \frac{1}{1-c^{\nu}}\right) \times \\
\left(y-\left(x c^{k}+t c^{j}\right)\right)^{k-1} \chi\left(x c^{k}+t c^{j}<y<y m a x\right. \\
\left(y+y_{m a x}\right.
\end{gathered}
$$

For numerical purposes we of course usually choose $y_{\min }=(x+t) c^{k}$ and $y_{\max }=x c^{k}+t$, and if $n+1<k-n$ we use the sum from 0 to $n$ while if $n+1>k-n$ we use the sum from $n+1$ to $k$. If $n+1=k-n$ there does not seem to be a reason to prefer one sum over the other.

The first formulation of Theorem 2, with $y_{\min }=x c^{k}$, is a direct consequence of (8.9). The second formulation, and formulations with more general $y_{\min }$ and $y_{\max }$, then follow from Lemma 5 above.

\section{Moments, from Theorem 2}

We define:

$$
\mu(x, t, k, r)=E\left[(X(t))^{r} \mid X(0)=x, N(t)=k\right]
$$


and

$$
\nu(x, t, k, r)=E\left[(X(t))^{r} \chi(N(t)=k) \mid X(0)=x\right]=\frac{(\lambda t)^{k}}{k !} e^{-\lambda t} \mu(x, t, k, r) .
$$

In theorem 2 , if we choose $x=0, y_{\min }=0$ we get for $k \geq 1$

$$
\begin{gathered}
E\left[(Y(t))^{r} \chi(N(t)=k)\right]= \\
\frac{e^{-\lambda t} \lambda^{k}}{(k-1) !} \sum_{j=0}^{k}(-1)^{j} c^{\frac{1}{2} j(j+1)} c^{-k j}\left(\prod_{\nu=1}^{j} \frac{1}{1-c^{\nu}}\right)\left(\prod_{\nu=1}^{k-j} \frac{1}{1-c^{\nu}}\right) \times \\
\int_{x c^{k}}^{x c^{k}+t c^{j}}\left(y-x c^{k}\right)^{r}\left(x c^{k}+t c^{j}-y\right)^{k-1} d y= \\
e^{-\lambda t} t^{r} \frac{(\lambda t)^{k}}{(r+1)(r+2) \cdots(r+k)} \sum_{j=0}^{k}(-1)^{j} c^{\frac{1}{2}(j-1) j} c^{(r+1) j}\left(\prod_{\nu=1}^{j} \frac{1}{1-c^{\nu}}\right)\left(\prod_{\nu=1}^{k-j} \frac{1}{1-c^{\nu}}\right)= \\
e^{-\lambda t} t^{r} \frac{(\lambda t)^{k}}{(r+1)(r+2) \cdots(r+k)} \frac{\left(1-c^{r+1}\right)\left(1-c^{r+2}\right) \cdots\left(1-c^{r+k}\right)}{(1-c)\left(1-c^{2}\right) \cdots\left(1-c^{k}\right)}
\end{gathered}
$$

The last step uses Lemma A.1 in appendix A.

We find that the same formula holds for $k=0$. Thus we have proven

\section{Theorem 3}

$$
\begin{gathered}
\nu(0, t, k, r)=E\left[(Y(t))^{r} \chi(N(t)=k)\right]= \\
e^{-\lambda t} \frac{\lambda^{k} t^{k+r} \Gamma(r+1)}{\Gamma(r+k+1)} \frac{\left(1-c^{r+1}\right)\left(1-c^{r+2}\right) \cdots\left(1-c^{r+k}\right)}{(1-c)\left(1-c^{2}\right) \cdots\left(1-c^{k}\right)}, \\
\mu(0, t, k, r)=E\left[(Y(t))^{r} \mid N(t)=k\right]= \\
\Gamma(r+1) t^{r} \frac{k !}{\Gamma(k+r+1)} \frac{\left(1-c^{r+1}\right)\left(1-c^{r+2}\right) \cdots\left(1-c^{r+k}\right)}{(1-c)\left(1-c^{2}\right) \cdots\left(1-c^{k}\right)} .
\end{gathered}
$$

It is easily seen that if $t \uparrow \infty, k \uparrow \infty$, while $\frac{k}{t} \rightarrow \lambda$, indeed $\mu(0, t, k, r)$ converges to $E\left[(X(\infty))^{r}\right]$ as in (1.6).

(10.4) also gives

$$
E\left[(Y(t))^{r} c^{\beta N(t)}\right]=\sum_{j=0}^{\infty} \nu(0, t, j, r) c^{\beta j}=
$$




$$
\Gamma(r+1) t^{r} e^{-\lambda t} \sum_{j=0}^{\infty} \frac{\left(\lambda t c^{\beta}\right)^{j}}{\Gamma(j+r+1)} \frac{\left(1-c^{r+1}\right)\left(1-c^{r+2}\right) \cdots\left(1-c^{r+j}\right)}{(1-c)\left(1-c^{2}\right) \cdots\left(1-c^{j}\right)} .
$$

For $r=k$, integer (and non-negative), we already had (3.17). We observe that (10.6) reduces to (3.17) if $r$ is a non-negative integer.

If $r$ is a non-negative integer then (for later use we write the finite binomial sum, $j$ from 0 to $r$, as an infinite sum) we have

$$
\begin{gathered}
\mu(x, t, k, r)=E\left[\left(Y(t)+x c^{N(t)}\right)^{r} \mid N(t)=k\right]= \\
\sum_{j=0}^{\infty}\left(\begin{array}{c}
r \\
j
\end{array}\right)\left(x c^{k}\right)^{r-j} \mu(0, t, k, j)= \\
\Gamma(r+1)\left(x c^{k}\right)^{r} \sum_{j=0}^{\infty} \frac{\left(x c^{k}\right)^{-j} t^{j}}{\Gamma(r-j+1)} \frac{k !}{(k+j) !} \frac{\left(1-c^{j+1}\right)\left(1-c^{j+2}\right) \cdots\left(1-c^{j+k}\right)}{(1-c)\left(1-c^{2}\right) \cdots\left(1-c^{k}\right)}= \\
\left(x c^{k}\right)^{r} \sum_{j=0}^{\infty} \frac{r(r-1) \cdots(r-j+1)}{(k+1)(k+2) \cdots(k+j)}\left(\frac{t}{x c^{k}}\right)^{j} \frac{\left(1-c^{j+1}\right)\left(1-c^{j+2}\right) \cdots\left(1-c^{j+k}\right)}{(1-c)\left(1-c^{2}\right) \cdots\left(1-c^{k}\right)},
\end{gathered}
$$

If $r$ is not a non-negative integer (10.7) becomes an infinite sum and we have to check when it converges and when it is an identity.

For (10.7) to be valid it is sufficient that $P\left\{0 \leq Y(t)<x c^{k}\right\}=1$. Since $0 \leq Y(t) \leq t$ it therefore is sufficient that $0 \leq t<x c^{k}$. In fact, (10.7) converges absolutely as long as $0 \leq t \leq x c^{k}$ (we leave this as an exercise for the reader). By the material in Appendix B (10.7) then actually holds as long as either $r$ is a non-negative integer (finite sum) or $0 \leq t \leq x c^{k}$ (absolutely converging sum).

A similar argument can be made if $0 \leq x c^{k} \leq t$. Since $0 \leq x c^{k}<t$ does not guarantee that $0 \leq x c^{k}<Y(t)$, this argument depends more heavily on the material in Appendix B. Nevertheless, we have that as long as either $r$ is a non-negative integer (finite sum) or $0 \leq x c^{k} \leq t$ (absolutely converging sum) then

$$
\mu(x, t, k, r)=E\left[\left(Y(t)+x c^{N(t)}\right)^{r} \mid N(t)=k\right]=
$$




$$
\begin{gathered}
\sum_{j=0}^{\infty}\left(\begin{array}{c}
r \\
j
\end{array}\right)\left(x c^{k}\right)^{j} \mu(0, t, k, r-j)= \\
\Gamma(r+1) t^{r} \sum_{j=0}^{\infty} \frac{\left(x c^{k}\right)^{j} t^{-j}}{j !} \frac{k !}{\Gamma(k+r-j+1)} \frac{\left(1-c^{r-j+1}\right)\left(1-c^{r-j+2}\right) \cdots\left(1-c^{r-j+k}\right)}{(1-c)\left(1-c^{2}\right) \cdots\left(1-c^{k}\right)}= \\
\frac{k ! \Gamma(r+1)}{\Gamma(r+k+1)} t^{r} \times \\
\sum_{j=0}^{\infty} \frac{(r+k)(r+k-1) \cdots(r+k-j+1)}{j !}\left(\frac{x}{t}\right)^{j} \frac{\left(c^{j}-c^{r-j+1}\right)\left(c^{j}-c^{r-j+2}\right) \cdots\left(c^{j}-c^{r-j+k}\right)}{(1-c)\left(1-c^{2}\right) \cdots\left(1-c^{k}\right)}
\end{gathered}
$$

If $r$ is a non-negative integer then (10.7) and (10.8) both are finite sums, and they are identical.

If $0 \leq x \leq t$ then $0 \leq x c^{k} \leq t$ for all $k \geq 0$. Thus, for $0 \leq x \leq t$ we can multiply (10.8) with $P\{N(t)=k\}$ and sum over $k$, thus obtaining that as long as either $r$ is a non-negative integer or $0 \leq x \leq t$ we have

$$
\begin{gathered}
E\left[(X(t))^{r} c^{\beta N(t)} \mid X(0)=x\right]= \\
\Gamma(r+1) t^{r} e^{-\lambda t} \sum_{j=0}^{\infty} \frac{(x / t)^{j}}{j !} \sum_{\nu=0}^{\infty} \frac{\left(c^{j} \lambda t c^{\beta}\right)^{\nu}}{\Gamma(\nu+r-j+1)} \frac{\left(1-c^{r-j+1}\right)\left(1-c^{r-j+2}\right) \cdots\left(1-c^{r-j+\nu}\right)}{(1-c)\left(1-c^{2}\right) \cdots\left(1-c^{\nu}\right)}
\end{gathered}
$$

If $r$ is a non-negative integer the outer sum in (10.9) is a finite sum ( $j$ from 0 to $r$ ) and is identical to (3.12). If $r$ is not a non-negative integer but $0 \leq x \leq t$ then (10.9) is an absolutely converging infinite sum and still is an identity. The latter statement is left as an exercise for the reader.

If $0<t \leq x$ we still have that $0 \leq x c^{k}<t$ for $k$ sufficiently large. Therefore, we can not use (10.7) the same way we used (10.8). It might be possible to obtain useful results by splitting the sum into the ranges $k<\frac{\log (x)-\log (t)}{|\log (c)|}$ and $k \geq \frac{\log (x)-\log (t)}{|\log (c)|}$. We have not tried this. 


\section{A Some auxiliary results}

Lemma A.1. For all $k \in\{0,1, \cdots\}$ and all $a$ :

$$
\frac{(1-a)(1-c a)\left(1-c^{2} a\right) \cdots\left(1-c^{k-1} a\right)}{(1-c)\left(1-c^{2}\right)\left(1-c^{3}\right) \cdots\left(1-c^{k}\right)}=\sum_{j=0}^{k}(-1)^{j} c^{\frac{1}{2}(j-1) j} a^{j} \prod_{\nu=1}^{j} \frac{1}{1-c^{\nu}} \prod_{\nu=1}^{k-j} \frac{1}{1-c^{\nu}}
$$

Proof A classical result in Hypergeometric Series (see e.g. [42] pp 7, 8 for a proof and historical notes) is that for $|z|<1,|c|<1$ and $a$ arbitrary

$$
\prod_{k=0}^{\infty} \frac{1-c^{k} a z}{1-c^{k} z}=\sum_{k=0}^{\infty}\left(\prod_{j=0}^{k-1} \frac{1-c^{j} a}{1-c^{j+1}}\right) z^{k}
$$

We also know (see [2] for a simple proof or [42] for more background information) that for $|c|<1$,

$$
\prod_{k=0}^{\infty} \frac{1}{1+c^{k} z}=\sum_{k=0}^{\infty} \frac{(-1)^{k} z^{k}}{(1-c)\left(1-c^{2}\right) \ldots\left(1-c^{k}\right)}
$$

and

$$
\prod_{k=0}^{\infty}\left(1+c^{k} z\right)=\sum_{k=0}^{\infty} \frac{c^{\frac{1}{2}(k-1) k} z^{k}}{(1-c)\left(1-c^{2}\right) \ldots\left(1-c^{k}\right)} .
$$

The last two results also give a powerseries for (A.2):

$$
\prod_{k=0}^{\infty} \frac{1}{1-c^{k} z} \prod_{k=0}^{\infty}\left(1-c^{k} a z\right)=\sum_{k=0}^{\infty} z^{k} \sum_{j=0}^{k}(-1)^{j} c^{\frac{1}{2}(j-1) j} a^{j} \prod_{\nu=1}^{j} \frac{1}{1-c^{\nu}} \prod_{\nu=1}^{k-j} \frac{1}{1-c^{\nu}} .
$$

Equating coefficients in the powerseries proves the result.

(A.1) shows that

$$
\begin{gathered}
\sum_{j=0}^{k}(-1)^{j} c^{\frac{1}{2}(j-1) j} a^{j} \prod_{\nu=1}^{j} \frac{1}{1-c^{\nu}} \prod_{\nu=1}^{k-j} \frac{1}{1-c^{\nu}}= \\
\begin{cases}\delta_{0, k} & \text { if } a=1=c^{0} \\
0 & \text { if } k \geq 1 \text { and } a=c^{-\mu}, \mu \in\{0,1,2, \cdots, k-1\} \\
\prod_{j=1}^{k-1} \frac{1-c^{\mu+j}}{1-c^{j}} & \text { if } a=c^{\mu}, \mu \in\{1,2, \cdots\} \\
>0 & \text { if }-1<c<+1 \text { and }-1<a<+1 .\end{cases}
\end{gathered}
$$


If $0<c<1$ we also know that the expression in (A.1) is positive for all $-\infty<a<1$.

With the Binomial Formula, the second case in (A.6) shows that if $k, \mu$, and $\kappa$ are integers with $1 \leq \mu \leq k$ and $0 \leq \kappa \leq \mu-1$ then

$$
\sum_{j=0}^{k}(-1)^{j} c^{\frac{1}{2} j(j+1)} c^{-\mu j}\left(\prod_{\nu=1}^{j} \frac{1}{1-c^{\nu}} \prod_{\nu=1}^{k-j} \frac{1}{1-c^{\nu}}\right)\left(r c^{j}+\beta\right)^{\kappa}=0 \text { for all } r, \beta .
$$

This proves the lemma in Section 9.

Lemma A.2 For all $|c|<1$, all $z$ and all $\kappa, k \in\{0,1,2, \cdots\}$ with $\kappa \leq k$

$$
\begin{gathered}
\sum_{\mu=0}^{k}(-1)^{\mu} c^{\frac{1}{2} \mu(\mu-1)-\mu(\kappa-1)} e^{z c^{\mu}}\left(\prod_{\nu=1}^{\mu} \frac{1}{1-c^{\nu}}\right)\left(\prod_{\nu=1}^{k-\mu} \frac{1}{1-c^{\nu}}\right)= \\
\sum_{j=\kappa}^{\infty} \frac{z^{j}}{j !} \prod_{\nu=1}^{k} \frac{1-c^{j-\kappa+\nu}}{1-c^{\nu}}= \\
\sum_{j=0}^{\infty} \frac{z^{j+\kappa}}{(j+\kappa) !} \prod_{\nu=1}^{k} \frac{1-c^{j+\nu}}{1-c^{\nu}} .
\end{gathered}
$$

Proof: The result trivially holds if $k=\kappa=0$. If $k \geq 1$, write, in the LHS, $e^{z c^{\mu}}$ as powerseries in $z c^{\mu}$, change order of summation, and use (A.6) and (A.1).

Similar results can of course be obtained if $e^{z c^{\mu}}$ is replaced by $f\left(z c^{\mu}\right)$, where $f($.$) can$ be written as a powerseries.

Somewhat similar results are

Lemma A.3

$$
\begin{gathered}
\sum_{\nu=0}^{\infty} \frac{c^{\beta \nu} e^{\lambda x c^{\nu}}}{(1-c) \cdots\left(1-c^{\nu}\right)}=\sum_{k=0}^{\infty} \frac{(\lambda x)^{k}}{k !} \sum_{\nu=0}^{\infty} \frac{c^{(\beta+k) \nu}}{(1-c) \cdots\left(1-c^{\nu}\right)}= \\
\left(\prod_{\nu=0}^{\infty} \frac{1}{1-c^{\beta+\nu}}\right) \sum_{k=0}^{\infty} \frac{(\lambda x)^{k}}{k !}\left(1-c^{\beta}\right) \cdots\left(1-c^{k-1+\beta}\right),
\end{gathered}
$$

Lemma A.4

$$
e^{\lambda x c^{\nu}} \gamma\left(r+2, \lambda x c^{\nu}\right)=\left(\lambda x c^{\nu}\right)^{r+2} \int_{0}^{1} s^{r+2-1} e^{(1-s) \lambda x c^{\nu}} d s=
$$




$$
\begin{gathered}
\left(\lambda x c^{\nu}\right)^{r+2} \int_{0}^{1} s^{r+2-1} \sum_{n=0}^{\infty} \frac{(1-s)^{n}\left(\lambda x c^{\nu}\right)^{n}}{n !} d s=\sum_{n=0}^{\infty} \frac{\left(\lambda x c^{\nu}\right)^{r+2+n}}{n !} B(r+2, n+1)= \\
\sum_{n=0}^{\infty} \frac{\left(\lambda x c^{\nu}\right)^{r+2+n}}{(r+2) \cdots(r+2+n)},
\end{gathered}
$$

and Lemma A.5

$$
\begin{gathered}
e^{\lambda x c^{\nu}} \int_{0}^{\lambda x c^{\nu}}(\log (u)) e^{-u} d u= \\
\left(e^{\lambda x c^{\nu}}-1\right) \log \left(\lambda x c^{\nu}\right)-\sum_{k=1}^{\infty} \frac{\left(\lambda x c^{\nu}\right)^{k}}{k !} \sum_{n=1}^{k} \frac{1}{n} .
\end{gathered}
$$

The following is often useful to rewrite expressions as occur in this paper:

Observation A.1 If $m$ and $n$ are non-negative integers then

$$
\frac{\left(1-c^{m+1}\right) \cdots\left(1-c^{m+n}\right)}{(1-c) \cdots\left(1-c^{n}\right)}=\frac{\left(1-c^{n+1}\right) \cdots\left(1-c^{n+m}\right)}{(1-c) \cdots\left(1-c^{m}\right)} .
$$

This observation is easily verified by considering the two cases $(m \leq n)$ and $(m \geq n)$.

By changing the sign of $z$ in (A.4) we obtain

$$
\prod_{j=0}^{\infty}\left(1-c^{j} z\right)=\sum_{j=0}^{\infty}(-1)^{j} \frac{c^{\frac{1}{2}(j-1) j} z^{j}}{(1-c)\left(1-c^{2}\right) \ldots\left(1-c^{j}\right)} .
$$

which we recognize as the limit of (A.1) for $k \rightarrow \infty$. By differentiating (A.13) with respect to $z$ we obtain

\section{Observation A.2}

$$
\left(\sum_{j=0}^{\infty} \frac{c^{j}}{1-c^{j} z}\right)\left(\prod_{j=0}^{\infty}\left(1-c^{j} z\right)\right)=\sum_{j=1}^{\infty} \frac{j(-1)^{j-1} c^{\frac{1}{2}(j-1) j} z^{j-1}}{(1-c) \cdots\left(1-c^{j}\right)}
$$

\section{B Moments and Powerseries}

Suppose we have a function $f($.$) which has the powerseries$

$$
f(x)=\sum_{j=0}^{\infty} \frac{f^{(j)}(a)}{k !}(x-a)^{j}
$$


with radius of convergence $R>0$. Suppose $X$ is a random variable. When can we conclude that

$$
E[f(X)]=\sum_{j=0}^{\infty} \frac{f^{(j)}(a)}{j !} E\left[(X-a)^{j}\right] ?
$$

Clearly, a sufficient condition is that there exists a $0<r<R$ with $P\{|X-a|<r\}=1$. However, (B.2) often holds even if $P\{|X-a|>R\}>0$. In that case, to prevent trivial exceptions, we always assume that $E\left[|X-a|^{j}\right]<\infty$ for all $j \geq 0$.

It is well known that as long as $f^{(n+1)}($.$) exists and is continuous over the necessary$ range,

$$
f(x)=\sum_{j=0}^{n} \frac{f^{(j)}(a)}{j !}(x-a)^{j}+\int_{a}^{x} \frac{(x-u)^{n}}{n !} f^{(n+1)}(u) d u
$$

therefore

$$
E[f(X)]=\sum_{k=0}^{n} \frac{f^{(k)}(a)}{k !} E\left[(X-a)^{k}\right]+E\left[\int_{a}^{X} \frac{(X-u)^{n}}{n !} f^{(n+1)}(u) d u\right] .
$$

Under the conditions above, a necessary and sufficient condition for (B.2) to hold is that

$$
\lim _{n \rightarrow \infty} E\left[\int_{a}^{X} \frac{(X-u)^{n}}{n !} f^{(n+1)}(u) d u\right]=0 .
$$

We consider the special case $f(x)=(C+x)^{r}=C^{r}\left(1+\frac{x}{C}\right)^{r}=x^{r}\left(1+\frac{C}{x}\right)^{r}$ with $C>0$.

We see that as long as $E\left[X^{j}\right]<\infty$ for all $j \geq 0$

$$
E\left[(C+X)^{r}\right]=\sum_{j=0}^{\infty}\left(\begin{array}{c}
r \\
j
\end{array}\right) C^{r-j} E\left[X^{j}\right]
$$

if and only if

$$
\lim _{n \rightarrow \infty}\left(\begin{array}{c}
r \\
n
\end{array}\right)(r-n) E\left[\int_{0}^{X / C}\left(\frac{X}{C}-u\right)^{n}(1+u)^{r-(n+1)} d u\right]=0,
$$

while as long as $E\left[X^{r-j}\right]<\infty$ for all $j \geq 0$

$$
E\left[(C+X)^{r}\right]=\sum_{j=0}^{\infty}\left(\begin{array}{l}
r \\
j
\end{array}\right) E\left[X^{r-j}\right] C^{j}
$$


if and only if

$$
\lim _{n \rightarrow \infty}\left(\begin{array}{c}
r \\
n
\end{array}\right)(r-n) E\left[X^{r} \int_{0}^{C / X}\left(\frac{C}{X}-u\right)^{n}(1+u)^{r-(n+1)} d u\right]=0 .
$$

If $P\{X \geq 0\}=1$, (B.6) holds as long as

$$
\lim _{n \rightarrow \infty}\left(\begin{array}{c}
r \\
n+1
\end{array}\right) E\left[\left(\frac{X}{C}\right)^{n+1}\right]=0
$$

(take $n+1>r$ ). If $P\{X>0\}=1$, (B.8) holds as long as

$$
\lim _{n \rightarrow \infty}\left(\begin{array}{c}
r \\
n+1
\end{array}\right) E\left[\left(\frac{C}{X}\right)^{r-(n+1)}\right]=0
$$

$($ take $n+1>r)$.

The upshot is that as long as $C>0$ and $X$ is a non-negative random variable, each of (B.6), (B.8) holds as long as the series in the Right Hand Side converges.

Moreover, we know that if $C>0$ and $X$ is a non-negative random variable and $n+1 \geq r$ then

$$
\left|E\left[(C+X)^{r}\right]-\sum_{j=0}^{n}\left(\begin{array}{c}
r \\
j
\end{array}\right) C^{r-j} E\left[X^{j}\right]\right| \leq\left|\left(\begin{array}{c}
r \\
n+1
\end{array}\right) C^{r-(n+1)} E\left[X^{n+1}\right]\right|
$$

while if $C>0$ and $X$ is a positive random variable and $n+1 \geq r$ then

$$
\left|E\left[(C+X)^{r}\right]-\sum_{j=0}^{n}\left(\begin{array}{c}
r \\
j
\end{array}\right) C^{j} E\left[X^{r-j}\right]\right| \leq\left|\left(\begin{array}{c}
r \\
n+1
\end{array}\right) C^{n+1} E\left[X^{r-(n+1)}\right]\right|
$$




\section{References}

[1] Mathis, M., Semke, J. Mahdavi, J. and Ott, T.J. (1997)

The Macroscopic Behavior of the TCP Congestion Avoidance Algorithm.

Computer Communications Review 27 (3), pp 67 - 82 (July 1997).

[2] Ott, T.J., Kemperman, J.H.B., and Mathis, M. (1996)

The Stationary Behavior of Idealized TCP Congestion Behavior.

Available at www.teunisott.com/Papers .

[3] Ott, T.J. (1999)

ECN protocols and the TCP paradigm.

Available at www.teunisott.com/Papers .

Also presented at the Workshop on Modeling of Flows and Congestion Control Mechanisms, Ecole Normale Superieure, Paris, (Sept 2000).

[4] Ott, T.J. (2005)

Transport Protocols in the TCP Paradigm and their Performance.

Telecommunication Systems 30:4 pp 351-385, Springer Dec 2005.

Also available in www.teunisott.com/Papers.

[5] Ott, T.J. (2006)

Rate of Convergence for the "Square Root Formula" in the Internet transmission control protocol.

Advances in Applied Probability 38 no 4 (Dec 2006) pp 1132-1154.

Also available in www.teunisott.com/Papers.

[6] Ott, T.J. and Swanson, J. (2006)

Stationarity of some processes in Transport Protocols.

Presented at the IFIP WG 7.3 MAMA workshop, June 2006. A shortened version 
appeared in Perf.Eval.Rev. 34 no 3 (Dec 2006) pp 30 - 32.

An extended version is available in www.teunisott.com/Papers.

[7] Ott, T.J. and Swanson, J. (2007)

Asymptotic Behavior of a Generalized TCP Congestion Avoidance Algorithm.

J Appl Prob. 44, pp 618-635, Sept 2007.

(This paper fills in "weak convergence" details related to the Dec 2005 "TCP Paradigm" paper.

Also available in www.teunisott.com/Papers.

[8] Ott, T.J. (2007)

On the Ornstein-Uhlenbeck process with Delayed Feedback.

Work in progress.Among other things, this paper shows under what circumstances delay in the feedback loop makes the Ornstein-Uhlenbeck process unstable. The purpose of this paper is to study possible oscillatory behavior due to one RTT delay in the feedback in TCP/IP, in the situation of the "TCP Paradigm".

An almost current copy is available in www.teunisott.com/Papers.

[9] Löpker, A.H. and van Leeuwaarden, J.S.H. (2007)

Transient Moments of the Window Size in TCP.

Preprint. Available at www.win.tue.nl/ leeuwaa/paper14.html.

Also Technical Report 2007-19, ISSN 1389-2355, Eurandom, Eindhoven, 2007.

[10] Kelly, C.T. (2003)

Improving performance in high speed wide area networks.

ACM SigComm Computer Communication Review, 32(2):83-91, 2003.

[11] Kelly, C.T. (2004)

Engineering flow controls in the Internet.

PhD Thesis, Cambridge University, 2004. 
[12] Lakshman, T.V., and Madhow, U. (1997)

The Performance of TCP/IP for Networks with high Bandwidth-Delay products and random loss.

Trans on Netw 1997.

[13] Padhye, J., Firoiu, V., Towsley, D., and Kurose, J. (1998)

Modeling TCP throughput: A simple model and its empirical validation. proceedings ACM SigComm 1998.

[14] Padhye, J., Firoiu, V., Towsley, D., and Kurose, J. (2000). Modeling of TCP RENO performance: A simple model and its empirical validation.

IEEE/ACM Transactions on Networking 8(2):133-145, 2000.

[15] Misra, V., Gong, W.B., and Towsley, D. (1999)

Stochastic differential equation modeling and analysis of TCP-windowsize behavior. Proceedings of IFIP WG 7.3 Performance 1999.

[16] Altman, E., Avrachenkov, K. and Barakat, C. (2000) A Stochastic model of TCP/IP with stationary random loss. ACM SigComm Computer Communications Review 30(4):231-242, 2000.

[17] Altman, E., Avrachenkov, K., Barakat, C. and Nunez-Queija, R. (2001) TCP modeling in the presence of nonlinear window growth. Proceedings of ITC-17, 2001.

[18] Altman, E., Avrachenkov, K. and Barakat, C. (2002) TCP Network Calculus: the case of large delay-bandwidth product. Proceeding of IEEE Infocom 2002. 
[19] Altman, E., Avrachenkov, K., Barakat, C. Kherani, A.A. and Prabhu, B.J. (2004) Analysis of scalable TCP. proceedings IEEE HSNMC 2004 pp 51-62.

[20] Altman, E., Barakat, C. and Ramos,V.M. (2004) Analysis of AIMD protocols over paths with variable delay. Proceedings IEEE Infocom 2004.

[21] Altman, E., Jimenez, T. and Kofman, D. (2004) DPS queues with stationary ergodic service times and the performance of TCP in overload. Proceedings IEEE Infocom 2004.

[22] Marquez, R., Altman, E., and Sole-Alvarez, S. (2004) Modeling TCP and high speed TCP: a nonlinear extension to AIMD mechanisms. Proceedings IEEE HSNMC 2004 pp 132-143 (2004).

[23] Altman, E., Avrachenkov, K., Kherani, A.A. and Prabhu, B.J. (2005) Performance Analysis and Stochastic Stability of congestion control protocols. Proceedings of IEEE Infocom 2005.

[24] Altman, E., Avrachenkov, K., and Prabhu, B. (2005) Fairness in MIMD congestion control algorithms. Proceedings of IEEE Infocom 2005.

[25] Baccelli, F., McDonald, D., and Reynier, J. (2002) A mean-field model for multiple TCP connec tions through a buffer implementing RED. Performance Evaluation 49:77-97, 2002. Also Proceedings Performance 2002. 
[26] Baccelli, F., Chaintreau, F., de Vleeschauwer, D., and McDonald, D. (2004) A mean-fields analysis od short lived interacting TCP flows. Performance Evaluation 2004. Also Proceedings ACM Sigmetrics 2004.

[27] Dumas, V., Guillemin, F., and Robert, Ph. (2002)

A Markovian analysis of additive-increase, multiplicative decrease (AIMD) algorithms.

Adv Appl Prob 34(1):85-111, 2002.

[28] Guillemin, F., Robert, Ph., and Zwart, B. (2004)

AIMD algorithms and exponential functionals.

Ann Appl Prob, 14(1):90-117, 2004.

[29] Bohacek, S. (2003)

A stochastic model of TCP and fair video transmission.

Proceedings Infocom 2003.

[30] Budhiraja, A., Hernandez-Campos F., Kulkarni, V.G. and Smith, F.D. (2004)

Stochastic differential equation for TCP window size: Analysis and experimental validation.

Probability in the Engineering and Information Sciences 18:111-140, 2004.

[31] Misra, A. and Ott, T.J. (1999)

The window distribution of idealized TCP congestion avoidance with variable packet loss.

Proceedings of IEEE Infocom 1999 pp 1564-1572, 1999.

[32] Baras, J., Misra, A. and Ott, T.J. (1999)

The window distribution of of multiple TCPs with random loss queues.

Proceedings of Globecomm 1999. 
[33] Baras, J., Misra, A. and Ott, T.J. (2000)

Generalized TCP congestion avoidance and its effect on bandwidth sharing and variability.

Proceedings of Globecomm 2000.

[34] Baras, J., Misra, A. and Ott, T.J. (2000)

Using drop-biasing to stabilize the occupancy of random drop queues with TCP traffic.

Proceedings of ICCS 2000.

[35] Misra, A. and Ott, T.J. (2001)

Effect of exponential averaging on the variability of a RED queue.

Proceedings of ICC 2001.

[36] Misra, A. and Ott, T.J. (2001)

Jointly coordinating ECN and TCP for rapid adaptation to varying bandwidth.

Proceedings of Milcom 2001.

[37] Baras, J., Misra, A. and Ott, T.J. (2002)

Predicting bottleneck bandwidth sharing by generalized TCP flows.

Computer Networks 40(4):557-576, 2002.

[38] Misra, A. and Ott, T.J. (2003)

Performance sensitivity and fairness of ECN-aware "modified TCP".

Journal of Performance Evaluation (PEVA) 53(3):255-272, 2003. Also published as Lecture Notes in Computer Science (LNCS), editors Gregory, Conti, Campbell, Omidyar, Zukerman.

[39] Sally Floyd (1994)

TCP and explicit congestion notification, ACM CCR, 24(5), October 1994. 
[40] Ramakrishnan, K., Floyd, S. and Black, D. (2001) The addition of Explicit Congestion Notification (ECN) to IP.

IETF RFC 3168, Sept 2001.

[41] Floyd, S. (2006) Specifying Alternate Semantics for the Explicit Congestion Notification (ECN) field.

IETF RFC 4774, Nov 2006.

[42] Gasper, G. and Rahman, M. (1990)

Basic Hypergeometric Series,

Encyclopedia of Mathematics and its Applications, Cambridge University Press.

[43] Abramowitz, M. and Stegun, I.A. (1970)

Handbook of Mathematical Functions

National Bureau of Standards, ninth printing (1970). 